\title{
Near-infrared probing of embedded structures in starburst and Seyfert galaxies ${ }^{\star}$
}

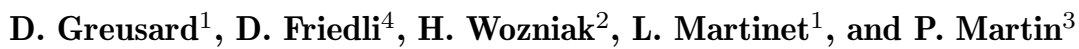 \\ 1 Observatoire de Genève, CH-1290 Sauverny, Switzerland \\ 2 Observatoire Astronomique de Marseille-Provence, F-13248 Marseille Cedex 4, France \\ 3 Canada-France-Hawaii Telescope, PO Box 1597, Kamuela, HI 96743, U.S.A. \\ ${ }^{4}$ Gymnase de Nyon, CH-1260 Nyon, Switzerland
}

Received March 14; accepted May 27, 2000

\begin{abstract}
Surface photometry in the $J$ and $K^{\prime}$ bands of 15 southern Seyfert or starburst galaxies is presented. The detailed central morphology and structural properties of these objects were analyzed by fitting ellipses to isophotes. New central peculiar structures have been identified like, for instance, three double-barred systems (ESO 215-G031; ESO 320-G030; ESO 443-G017), one object with a nested nuclear spiral structure at the center of a primary bar (NGC 5135), one object with a nuclear bar without evidence of a large-scale bar (NGC 4941), and one galaxy with a likely dissolved secondary bar within a primary one (ESO 508-G005). The $J-K^{\prime}$ radial profile proved to be reasonably well linked with the presence of a starburst, but not with the Seyfert activity. For significant starbursts, the central $J-K^{\prime}$ value is $0.3-1.5$ magnitude larger than the disc one.
\end{abstract}

Key words: galaxies: structure — galaxies: photometry — galaxies: Seyfert — galaxies: starburst — infrared: galaxies

\section{Introduction}

Fueling of the inner region of starburst galaxies and active galactic nuclei (AGN) is still an unresolved issue. Several clues are useful to investigate this problem. For example, Shlosman et al. (1989) have suggested that bars within bars are engines to include in mechanisms for gas fueling active nuclei. In this scenario, the primary (large-scale) bar brings gas, through angular momentum transfer, from

Send offprint requests to: D. Greusard, e-mail: Didier.Greusard@obs.unige.ch

* Based on observations collected at the European Southern Observatory, La Silla, Chile (ESO programme 59.A-0773). the outer part of the galaxy down to few kpc from the central region. From there, the nuclear bar could take over and drive this gas toward the inner region. Friedli \& Martinet (1993) using 3D self-consistent numerical simulations confirmed the possibility of forming stable systems (over several Gyr) with secondary bars within primary bars, and showed that these features might play a key role in collecting gas into the central region. Moreover, such embedded structures have already been observed in several galaxies (e.g. de Vaucouleurs 1974; Jarvis et al. 1998; Wozniak et al. 1995, Paper I).

Bringing material from the periphery to a region very close to the central activity requires a multi-scale process, so a multi-scale analysis appears appropriate. In addition, the fueling activity problem seems also to depend on the activity type (AGN or starburst):

- Primary bars (large-scale): contrary to other previous results (e.g. Ho et al. 1997), Knapen et al. (2000) suggest that Seyfert hosts are barred more often that nonSeyfert ones. Starburst galaxies seem to have a higher incidence of primary bars (e.g. Hunt \& Malkan 1999).

- Secondary bars (small-scale): it is not yet well established if they are more prevalent in Seyferts than in non-active galaxies (Paper I; Jungwiert et al. 1997) or not Mulchaey \& Regan (1997).

Thus additional morphological and kinematical studies of starburst and AGN remain necessary to give some more insight on the host dynamics and evolution.

In view of all previous considerations, this study presents $J$ and $K^{\prime}$ surface photometry of fifteen starburst and Seyfert galaxies in order to infer their morphological and structural properties. The principal aim is to detect bars and embedded bars among objects of this sample. The near-IR bands are less affected by dust than visual ones, and they are well suited to study the obscured central parts of disc galaxies. The information provided by these filters is essential to obtain reliable parameters 
Table 1. Sample of observed galaxies

\begin{tabular}{|c|c|c|c|c|c|c|c|c|c|}
\hline Galaxies & $\alpha(2000)$ & $\delta(2000)$ & $\begin{array}{c}\text { Type } \\
(4)\end{array}$ & $\begin{array}{c}z \\
{\left[10^{-3}\right]} \\
(5)\end{array}$ & $\begin{array}{c}D_{25} \\
{\left[{ }^{\prime}\right]} \\
(6)\end{array}$ & $\begin{array}{c}i \\
{\left[{ }^{\circ}\right]} \\
(7)\end{array}$ & $\begin{array}{c}\mathrm{PA}_{\text {disc }} \\
{\left[^{\circ}\right]} \\
(8)\end{array}$ & $\begin{array}{c}\text { Central } \\
\text { Activity } \\
(9)\end{array}$ & $\begin{array}{c}t_{\text {expo }} \\
{[\mathrm{s}]} \\
(10)\end{array}$ \\
\hline ESO 374-G032 & $10^{\mathrm{h}} 06^{\mathrm{m}} 04^{\mathrm{s}}$ & $-33^{\circ} 53^{\prime} 02^{\prime \prime}$ & GPair & 34 & & 51 & & STB & $1200 / 1200$ \\
\hline ESO 264-G036 & $10^{\mathrm{h}} 43^{\mathrm{m}} 08^{\mathrm{s}}$ & $-46^{\circ} 12^{\prime} 39^{\prime \prime}$ & $\mathrm{SB}(\mathrm{s}) \mathrm{b}$ & 23 & $1.1 \times 0.7$ & 49 & 102 & & $1200 / 1200$ \\
\hline NGC 3393 & $10^{\mathrm{h}} 48^{\mathrm{m}} 24^{\mathrm{s}}$ & $-25^{\circ} 09^{\prime} 40^{\prime \prime}$ & $\left(\mathrm{R}^{\prime}\right) \mathrm{SB}(\mathrm{s}) \mathrm{ab}$ & 12 & $2.2 \times 2.0$ & 24 & & STB/Sy2 & $1200 / 1200$ \\
\hline ESO 215-G031 & $11^{\mathrm{h}} 10^{\mathrm{m}} 35^{\mathrm{s}}$ & $-49^{\circ} 06^{\prime} 12^{\prime \prime}$ & $\left(\mathrm{R}^{\prime} 1\right) \mathrm{SB}(\mathrm{r}) \mathrm{b}$ & 9 & $2.4 \times 1.7$ & 41 & 130 & STB & $1200 / 1200$ \\
\hline ESO 320-G030 & $11^{\mathrm{h}} 53^{\mathrm{m}} 12^{\mathrm{s}}$ & $-39^{\circ} 07^{\prime} 49^{\prime \prime}$ & $\left(\mathrm{R}^{\prime} 1\right) \mathrm{SAB}(\mathrm{r}) \mathrm{a}$ & 11 & $2.2 \times 1.3$ & 55 & 121 & STB & $1200 / 1200$ \\
\hline ESO 443-G017 & $12^{\mathrm{h}} 57^{\mathrm{m}} 45^{\mathrm{s}}$ & $-29^{\circ} 45^{\prime} 59^{\prime \prime}$ & $(\mathrm{R}) \mathrm{SB}\left(\mathrm{r}^{\prime} \mathrm{l}\right) 0 / \mathrm{a}$ & 10 & $1.4 \times 0.9$ & 50 & 23 & STB & $1200 / 1200$ \\
\hline NGC 4903 & $13^{\mathrm{h}} 01^{\mathrm{m}} 23^{\mathrm{s}}$ & $-30^{\circ} 56^{\prime} 02^{\prime \prime}$ & $\mathrm{SB}(\mathrm{rs}) \mathrm{c}$ & 16 & $1.5 \times 1.3$ & 36 & 73 & Sy2 & $1200 / 1200$ \\
\hline NGC 4941 & $13^{\mathrm{h}} 04^{\mathrm{m}} 13^{\mathrm{s}}$ & $-05^{\circ} 33^{\prime} 06^{\prime \prime}$ & $(\mathrm{R}) \mathrm{SAB}(\mathrm{r}) \mathrm{ab}$ & 4 & $3.6 \times 1.9$ & 57 & 15 & Sy2 & $1200 / 1200$ \\
\hline NGC 4939 & $13^{\mathrm{h}} 04^{\mathrm{m}} 14^{\mathrm{s}}$ & $-10^{\circ} 20^{\prime} 23^{\prime \prime}$ & $\mathrm{SA}(\mathrm{s}) \mathrm{bc}$ & 10 & $5.5 \times 2.8$ & 59 & 10 & Sy2 & $1200 / 1200$ \\
\hline ESO 323-G077 & $13^{\mathrm{h}} 06^{\mathrm{m}} 27^{\mathrm{s}}$ & $-40^{\circ} 24^{\prime} 50^{\prime \prime}$ & $(\mathrm{R}) \mathrm{SB}(\mathrm{l}) 0$ & 15 & $1.5 \times 1.0$ & 47 & 155 & STB/Sy1 & $1200 / 1200$ \\
\hline ESO 508-G005 & $13^{\mathrm{h}} 06^{\mathrm{m}} 56^{\mathrm{s}}$ & $-23^{\circ} 55^{\prime} 02^{\prime \prime}$ & $\mathrm{SB}(\mathrm{rl}) 0 / \mathrm{a}$ & 10 & $1.3 \times 1.0$ & 41 & & Sy2 & $1200 / 1200$ \\
\hline NGC 5135 & $13^{\mathrm{h}} 25^{\mathrm{m}} 44^{\mathrm{s}}$ & $-29^{\circ} 50^{\prime} 02^{\prime \prime}$ & $\mathrm{SB}(\mathrm{l}) \mathrm{ab}$ & 14 & $2.6 \times 1.8$ & 45 & & STB/Sy2 & $1200 / 1200$ \\
\hline NGC 5643 & $14^{\mathrm{h}} 32^{\mathrm{m}} 41^{\mathrm{s}}$ & $-44^{\circ} 10^{\prime} 28^{\prime \prime}$ & $\mathrm{SAB}(\mathrm{rs}) \mathrm{c}$ & 4 & $4.6 \times 4.0$ & 29 & & Sy2 & $1200 / 600$ \\
\hline NGC 6221 & $16^{\mathrm{h}} 52^{\mathrm{m}} 47^{\mathrm{s}}$ & $-59^{\circ} 12^{\prime} 59^{\prime \prime}$ & $\mathrm{SB}(\mathrm{s}) \mathrm{bc}$ pec & 5 & $3.5 \times 2.5$ & 46 & 5 & Sy2 & $900 / 900$ \\
\hline NGC 6300 & $17^{\mathrm{h}} 16^{\mathrm{m}} 59^{\mathrm{s}}$ & $-62^{\circ} 59^{\prime} 11^{\prime \prime}$ & $\mathrm{SB}(\mathrm{rs}) \mathrm{b}$ & 4 & $4.5 \times 3.0$ & 49 & 118 & Sy2 & $900 / 750$ \\
\hline
\end{tabular}

Columns (2), (3), (4), (5), (6) \& (7): from the NASA/IPAC Extragalactic Database (NED).

Column (8): position angle on the sky measured from North through East; from de Vaucouleurs et al. (1991).

Column (9): Sy $=$ Seyferts from Véron \& Véron $(1993) / \mathrm{STB}=\operatorname{starbursts} \leftrightarrow \log \left(S_{60} / S_{100}\right) \gtrsim-0.35$ (see text).

Column (10): total exposure time, $x / y \rightarrow x$ seconds in $J$-band and $y$ seconds in $K^{\prime}$-band.

about the old stellar population which is dynamically the most important one. While several near-IR imaging studies have recently been published (Friedli et al. 1996, Paper II; Jungwiert et al. 1997; Mulchaey et al. 1997; Alonso-Herrero et al. 1998; Peletier et al. 1999; Márquez et al. 1999), the total number of galaxies observed in these wavelengths is still modest so new data are necessary. Moreover, since galaxies might experienced secular evolution (see Friedli 1999 for a review), i.e. the small and large-scale morphology of galaxies could change over cosmological time-scales ( $\approx 10 \mathrm{Gyr}$ ), it is crucial to observe the largest sample possible of various morphological configurations. Thus increasing appreciably near-IR data is essential to study the birth and evolution of embedded structures.

The paper is organized as follows. The sample, observations, reduction processes and the analysis methods are described in Sect. 2. Section 3 contains the results in form of individual descriptions of the sampled objects. The global analysis and discussions are given Sect. 4 and our conclusions in Sect. 5 .

\section{Observations, data reduction and analysis}

The sample of galaxies is presented in Table 1. All Seyfert 2 galaxies have been selected from the Véron's catalog Véron-Cetty \& Véron (1993) with the following criteria: $V$ magnitude less than 15 , not or weakly inter- acting, and not too inclined. Galaxies with an IRAS colour $\log \left(S_{60} / S_{100}\right) \gtrsim-0.35$ are considered as starbursts (see Sect. 3.2.2) and have been picked out from Rowan-Robinson \& Crawford (1989). Despite its "nonactivity", ESO264-G036 has also been observed because it has $\log \left(S_{60} / S_{100}\right) \approx-0.37$, and its starburst component defined in Rowan-Robinson \& Crawford (1989) suggests a transition case between starburst and non-starburst galaxies.

A visual inspection of these objects was performed to obtain the final sample. We do not make any attempt to make our selection criteria free of bias. For instance, our sample is obviously biased towards the search for nuclear structure.

\subsection{Observations and reductions}

The observations were carried out on April 7-9, 1997, with the ESO/MPI 2.2 m telescope at La Silla; the conditions were photometric with a FWHM seeing ranging from 1.2 to $1.6^{\prime \prime}$. The infrared imaging camera IRAC-2b was equipped with a $256 \times 256$ NICMOS- 3 array with a pixel size of 0.507 pixel $^{-1}$, giving a field of view of $129^{\prime \prime} \times 129^{\prime \prime}$. For each object, detector saturation was avoided by taking a series of exposures on the object interspersed with sky exposures. This procedure of alternating between the source and the sky was repeating until the total integration time in Table 1 was reached. 
Table 2. Aperture photometry comparison between our study and published magnitudes $\Delta J$ and $\Delta K^{\prime}$

\begin{tabular}{ccrrrr}
\hline \multicolumn{1}{c}{ Galaxies } & $\begin{array}{c}\text { Aperture } \\
{\left[{ }^{\prime}\right]}\end{array}$ & \multicolumn{1}{c}{$J_{\text {our }}$} & \multicolumn{2}{c}{$K_{\text {our }}^{\prime}$} & \multicolumn{1}{c}{$\Delta J$} \\
& 15 & 11.3 & 10.3 & 0.31 & 0.20 \\
\hline NGC 3393 (1) & $15 K^{\prime}$ & \\
& 30 & 10.8 & 9.8 & 0.31 & 0.16 \\
NGC 4941 (2) & 14 & 11.2 & 10.2 & -0.21 & -0.13 \\
NGC 5135 (3) & 12 & 11.3 & 10.0 & -0.07 & 0.06 \\
& 34 & 10.5 & 9.4 & -0.28 & -0.08 \\
NGC 5643 (4) & 34 & 10.2 & 9.1 & 0.03 & 0.07 \\
& 51 & 9.9 & 8.8 & 0.11 & 0.20 \\
NGC 6221 (5) & 34 & 9.8 & 8.7 & -0.09 & -0.03 \\
& 51 & 9.4 & 8.3 & -0.12 & -0.04 \\
\hline \hline Mean algebraic difference & & & 0.00 & +0.05 \\
Mean absolute difference & & & 0.17 & 0.11 \\
Root mean square deviation (rms) & & 0.21 & 0.12 \\
\hline
\end{tabular}

(1): from Alonso-Herrero et al. (1998);

(2): from Dultzin-Hacyan \& Benitez (1994);

(3), (4), (5): from Glass \& Moorwood (1985).

Each frame was cleaned from cosmic rays as well as from cold and hot pixels. Flat-field frames were obtained from exposures taken each night on a uniform illuminated blank screen (dome flat-field). After stars were removed from sky frames, the sky background was computed using the mean of the two sky frames gathered before and after a science frame. Galaxy frames were then sky subtracted and divided by the normalized dome flat-field.

As they follow the rapid variation of the sky structures, mean sky frames were preferred to median sky frames (median of all sky frames taken over the observation of one galaxy). As they are not affected by the intrinsic timedependent unflatness of the sky, dome flat-fields were preferred rather than median sky flat-fields (median of all sky frames taken over the night). These choices have been confirmed by the flatness of the images, the quality of the background substraction and the photometric calibration.

Finally, all frames of one galaxy were co-added to create a time-cumulated science frame. As the telescope was moved between two science expositions, galaxy frames had to be shifted with regard to reference points; they were defined as the peak of a Gaussian adjusted to the intensity profile of stars present in all frames of a same object.

\subsection{Photometric calibrations}

Frame calibration was achieved by observing infrared standards from Carter \& Meadows (1995) during each night (2 on April 7th and 9th, and 3 on April 8th). The zero points of each night are all compatible within their rms error. We have used the zero point obtained from the 3 nights put together; that way the rms error in the

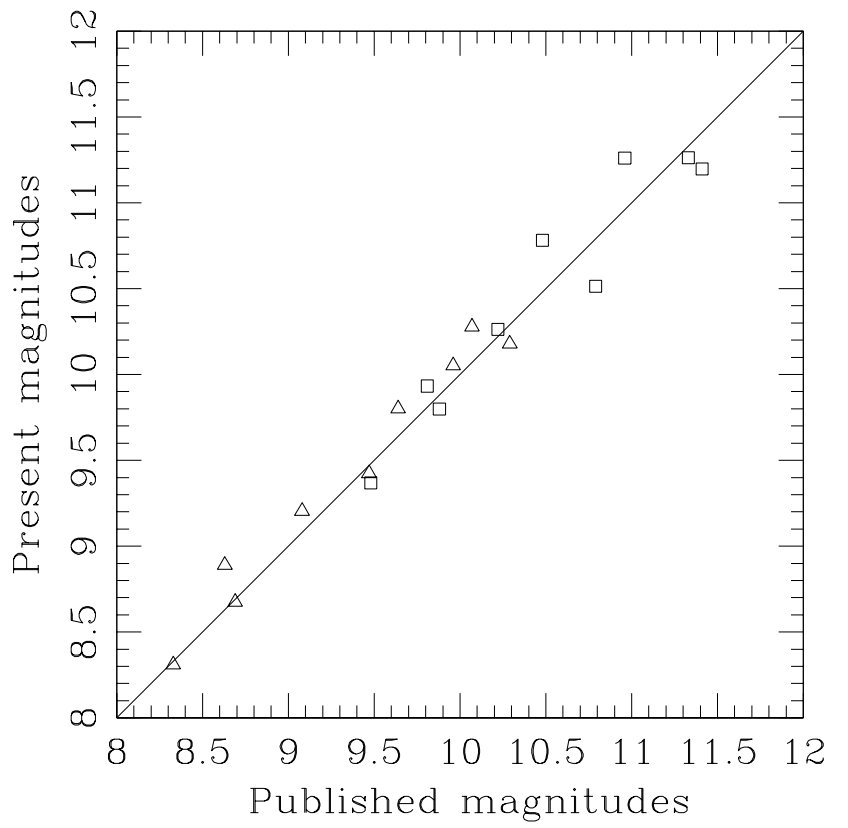

Fig. 1. Comparison with published data of aperture photometry for the objects listed in Table 2. Squares are for the $J$-band and triangles for the $K^{\prime}$-band

determination of the photometric zero points was $\approx 0.04$ $(J)$ and $\approx 0.03\left(K^{\prime}\right)$. In order to check our calibration, we have compared our results with aperture photometry taken from literature (see Table 2 and Fig. 1). Since the difference between $K^{\prime}$ and $K$ is weak and subject to uncertainties Wainscoat \& Cowie (1992), we have directly compared $K^{\prime}$ with $K$ photometry. The weak mean algebraic difference (see Table 2 ) reveals very little systematic deviation between our measurements and the published ones. The $K^{\prime}$ case may reflect the slight difference between $K^{\prime}$ and $K$; Wainscoat \& Cowie (1992) found that, for a sample of $16 \mathrm{~A}$ stars or $\mathrm{M}$ dwarfs, the $K^{\prime}$ filter has a zero point which is $0.03-0.04$ mag fainter than the $K$ one. But they also emphasize that this zero point departure may differ for a wider population of stars. Since there is very little systematic deviation and with regard to the mean absolute differences and rms, our calibrations are accurate enough in the context of this morphological study.

As the airmass correction obtained from mean atmospheric extinction coefficient for this site $\left(a_{J}=0.08\right.$, $a_{K^{\prime}}=0.11 \mathrm{mag} /$ airmass) does not improve our calibration check, it was not applied. No attempt was made to take the Galactic extinction and the $K$-correction into account.

\subsection{Surface photometry analysis}

Ellipses were fitted to isophotes of the whole sample in both $J$ and $K^{\prime}$ bands. As shown by various authors 
(e.g. Paper I), this technique provides good qualitative and quantitative estimations of the shape of embedded structures. As we want to observe the mean behaviour of isophotes, field stars and regions of intense emission (e.g. giant star forming regions) were flagged on galaxy frames before ellipse fitting. The program used is described in Paper I (and references therein). Each ellipse is characterized by:

- its semi-major axis length, $a\left[{ }^{\prime \prime}\right]$;

- its ellipticity $e, e=1-b / a$ where $b$ is the semi-minor axis;

- its intensity converted into surface brightness $\mu$, $\left[\operatorname{mag}^{\prime \prime-2}\right]$;

- its position angle, $\mathrm{PA}\left[^{\circ}\right]$, which is the angle between the semi-major axis and the North. This angle was defined to match the conventional notation: North is up, East at the left. Angles are measured from North and are positive in the anti-clockwise direction modulo $180^{\circ}$.

Thus, in each band, $\mu(a), e(a)$, and $\operatorname{PA}(a)$ profiles are derived, with $a$ increasing by a factor 1.01 between each point. In addition, "differential profiles" $\left(\mu_{J}-\mu_{K^{\prime}}, e_{J}-\right.$ $e_{K^{\prime}}$, and $\left.\mathrm{PA}_{J}-\mathrm{PA}_{K^{\prime}}\right)$ was computed as the difference between profiles in each band at the same semi-major axis. For that purpose individual profiles were linearly interpolate between each point.

\subsection{HST and literature data}

Because of their high spatial resolution, HST frames are useful to visually detect and/or confirm the presence of potential central asymmetries. Thus, when available, WFPC2 and/or NICMOS calibrated frames have been obtained from the Hubble Data Archive. The planetary camera CCD of WFPC2 instrument generally gave images of the galaxy centers in the $\mathrm{F} 606 \mathrm{~W}$ filter, with a pixel size 0 '.0455 and a field of view of $37^{\prime \prime} \times 37^{\prime \prime}$. NICMOS frames were gathered with the F160W filter $(\approx H$ band) and have a pixel size of $0 . \prime 075 /$ pixel and a field of view of $19^{\prime \prime} .2 \times$ 19 .'2.

In Sect. 3.2.2, where the behaviour of the NIR colour profile versus IRAS colour is studied, data from the literature have enriched our sample. Galaxies for which photometric $J, K^{\prime}$ frames and IRAS data were available were found in Alonso-Herrero et al. (1998) and in Paper II. Hence $J$ and $K^{\prime}$ frames (with different pixel sizes 0.286 pixel $^{-1}$ or 0 .' 143 pixel $^{-1}$ ) of 7 galaxies were obtained from the former study, as well as $J$ and $K$ frames (with different pixel sizes 0 '! 49 pixel $^{-1}, 0^{\prime \prime} 6$ pixel $^{-1}$, or 0 '.9 pixel $^{-1}$ ) of 5 galaxies from the latter. Ellipse fitting was performed on those galaxies in exactly the same way as for our sample.

\subsection{Terminology}

We use in this paper the following terminology:

- Primary bar: a bar with a scale-length of several kpc;

- Secondary bar: a bar with a scale-length around one kpc or less, and nested within a primary bar;

- Nuclear bar: the same feature as the secondary bar but with no evidence for a primary bar;

- $a_{\mathrm{p}}\left(a_{\mathrm{s}}\right), e_{\mathrm{p}}^{\max }\left(e_{\mathrm{s}}^{\max }\right), \mathrm{PA}_{\mathrm{p}}\left(\mathrm{PA}_{\mathrm{s}}\right)$ are respectively the length, the maximum ellipticity, and the position angle of the primary (secondary) bar;

- $\theta_{12}$ is the angle between the two bars. Positive values are for leading secondary bars whereas negative values are for trailing secondary bars (with respect to the primary bar);

$-\beta_{12} \equiv a_{\mathrm{p}} / a_{\mathrm{s}}$

- $\gamma_{12} \equiv L_{\mathrm{p}} / L_{\mathrm{s}}$ where $L_{\mathrm{p}}$ and $L_{\mathrm{s}}$ are the luminosity of the primary and secondary bars.

All the definitions above but the nuclear bar follow those of Papers I and II, and all the parameters have been computed in the same way as in Paper I.

\section{Results}

The set of Figs. 2 presents for each object in $J$ and $K^{\prime}-$ bands:

- the 2D image;

- the 1D profiles of surface brightness, ellipticity, and position angle, as a function of the semi-major axis length;

- the $J-K^{\prime}$ colour map;

- the differential profiles of surface brightness $\left(\mu_{J}-\mu_{K^{\prime}}\right)$, ellipticity $\left(e_{J}-e_{K^{\prime}}\right)$, and position angle $\left(\mathrm{PA}_{J}-\mathrm{PA}_{K^{\prime}}\right)$.

In addition, when available, HST NICMOS images are also shown in Figs. 2.

We hereafter describe the outstanding features and structural parameters of each galaxy, as they have been inferred from Figs. 2, HST images and from the literature. This presentation is not exhaustive; only the characteristics important in the context of this paper are emphasized. All quoted value of the structural parameters refer to the $K^{\prime}$-band unless otherwise specified. These parameters are summarized in Table 3 for galaxies with embedded structures. All linear distances have been computed assuming $H_{0}=75 \mathrm{~km} \mathrm{~s}^{-1} \mathrm{Mpc}^{-1}$.

\section{1. $E S O 374-G 032$ (Gpair, STB, $1^{\prime \prime} \approx 0.66 \mathrm{kpc}$ )}

The morphology of this galaxy shows evidence of recent or ongoing interaction (mainly a large tidal tail and a disturbed central feature). From the original IR frames, ESO 374-G032 shows at least two bright components within the central region separated by around 
ESO 374-G032
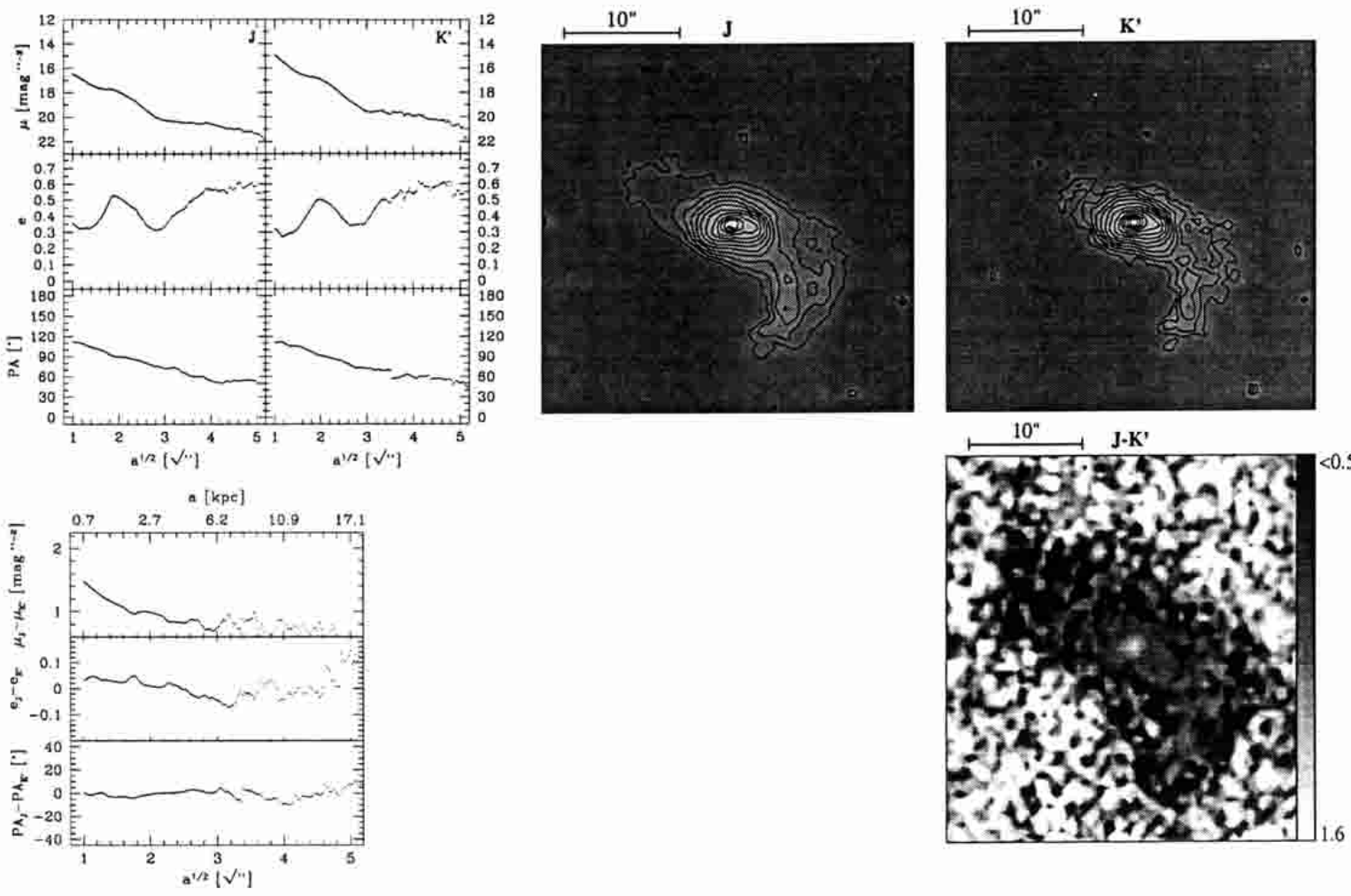

ESO 264-G036
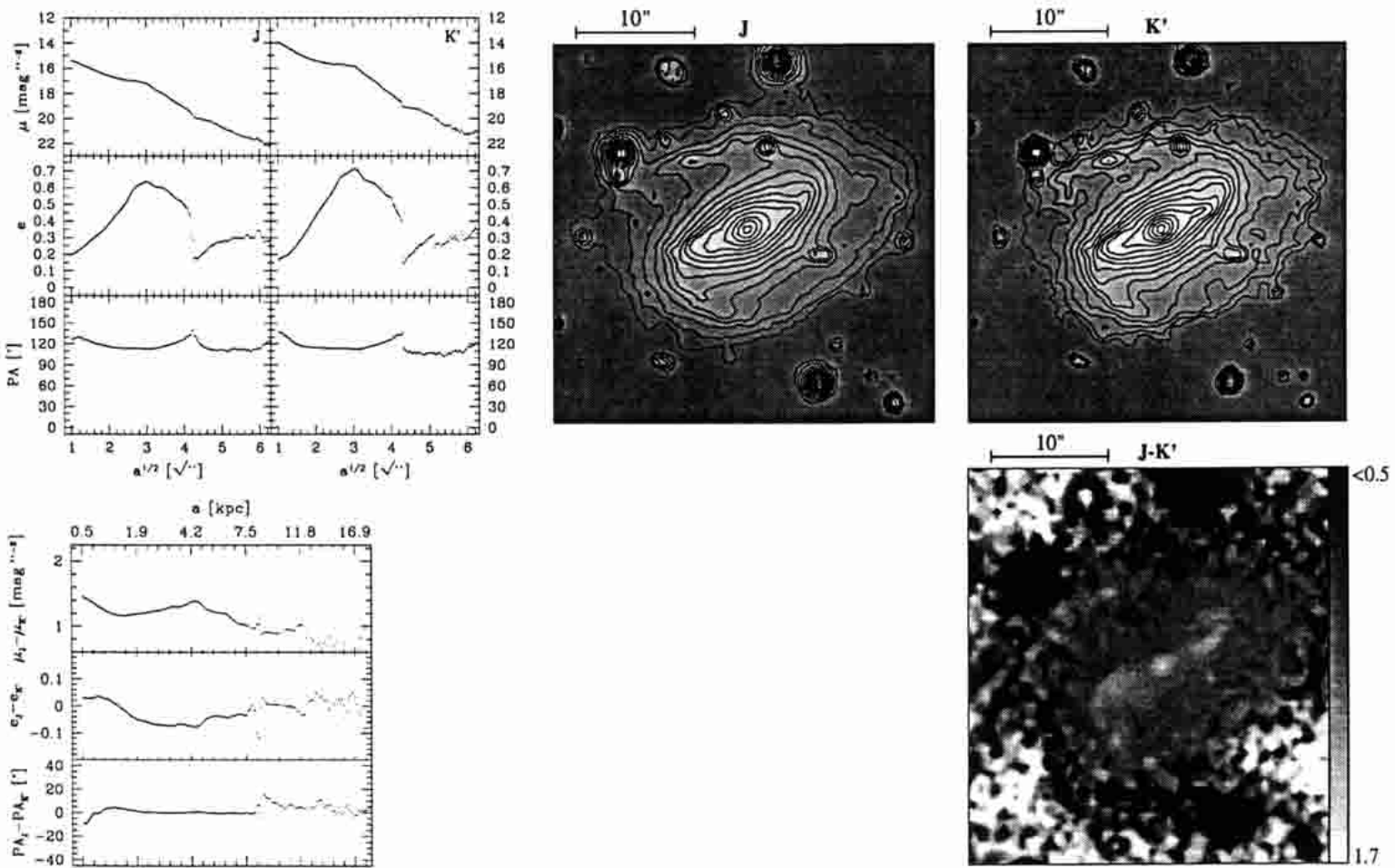

Fig. 2. For each of the fifteen galaxies, five (or six) panels are shown: Top left. Radial profiles of surface brightness $\mu$, ellipticity $e$, and position angle PA. $J$-band is on the left, $K^{\prime}$-band on the right. Note the square root scale. Top right. $J$ and $K^{\prime}$ grey-scale images with contours superimposed. The spacing is 0.4 mag. North is up, East on the left. Bottom left. $J-K^{\prime}$ radial profile. Bottom right. HST NICMOS F160W frame (when available) and $J-K^{\prime}$ colour map 
NGC 3393

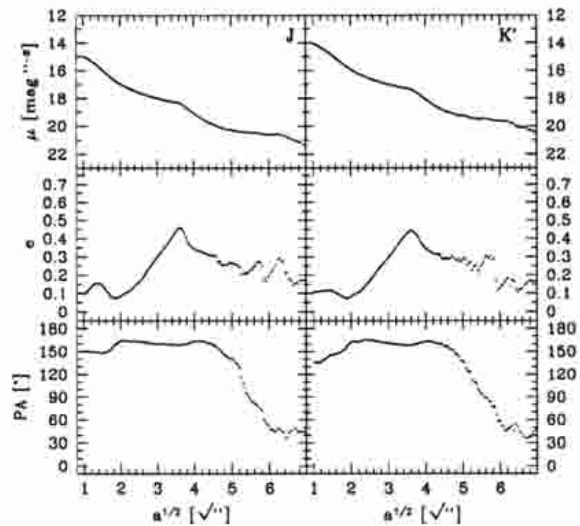

a [kpc]

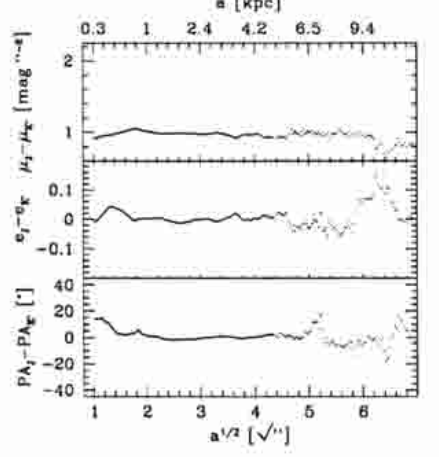

ESO 215-G031
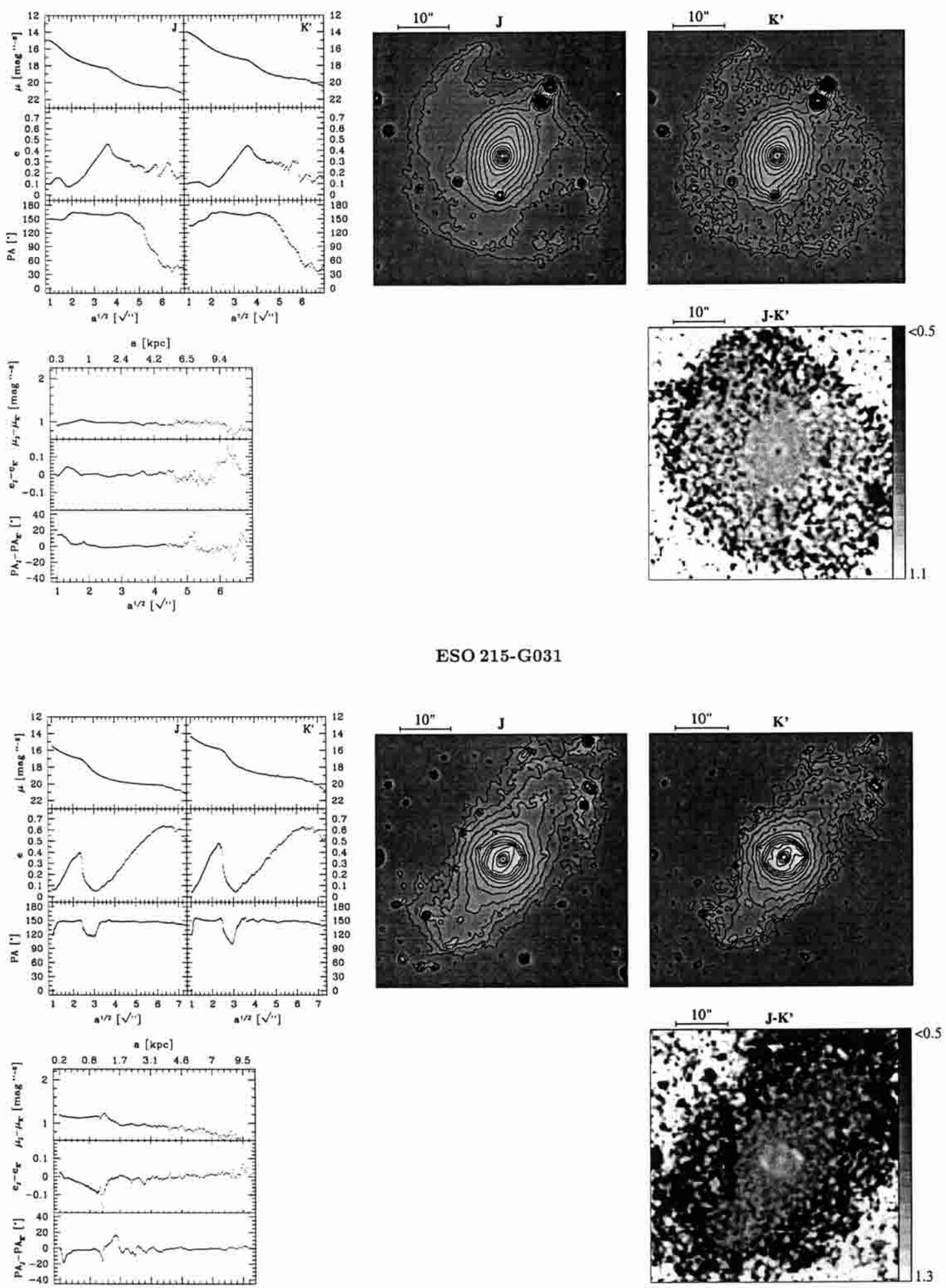

Fig. 2. continued 
ESO 320-G030
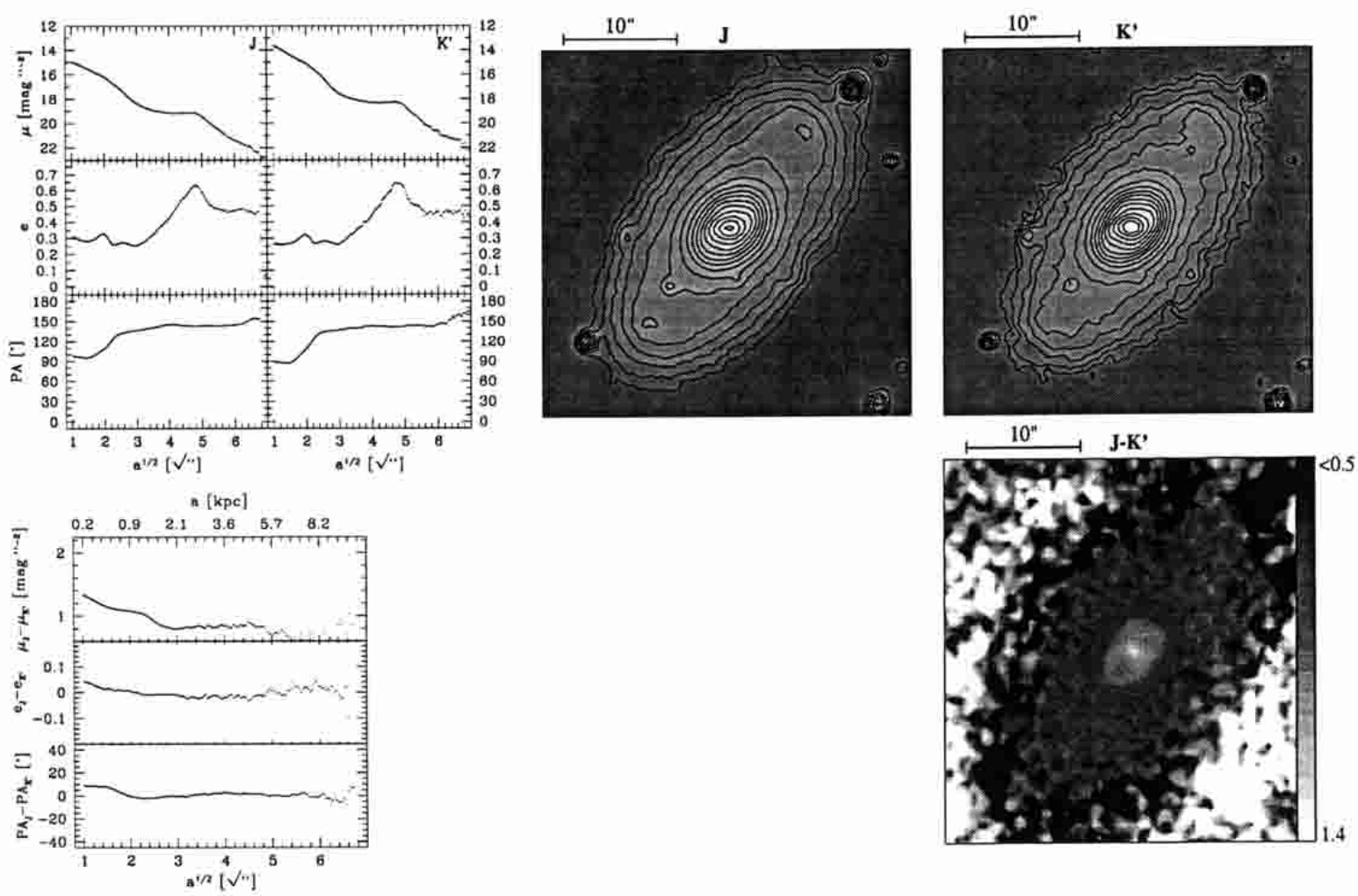

ESO 443-G017
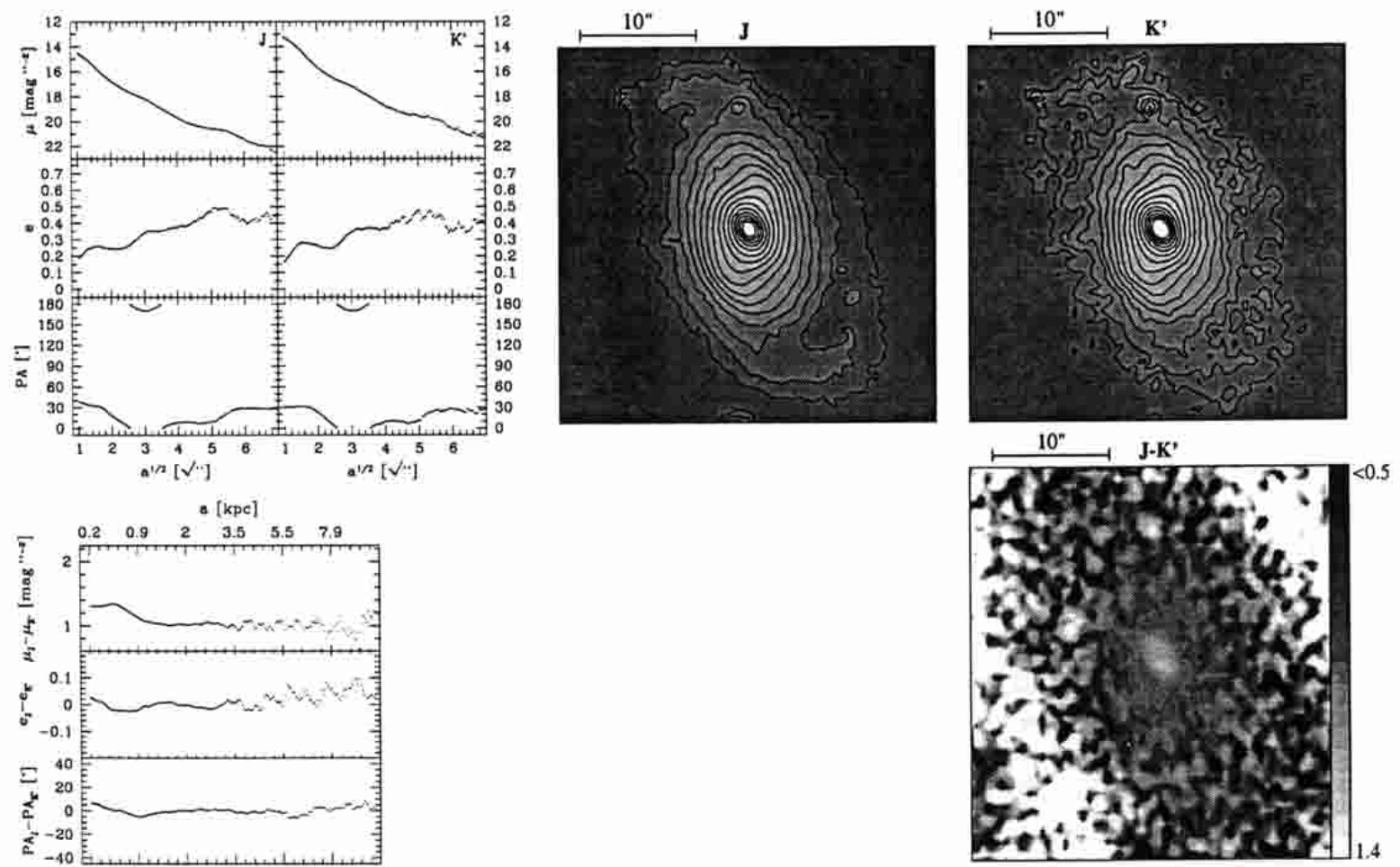

Fig. 2. continued 
NGC 4903
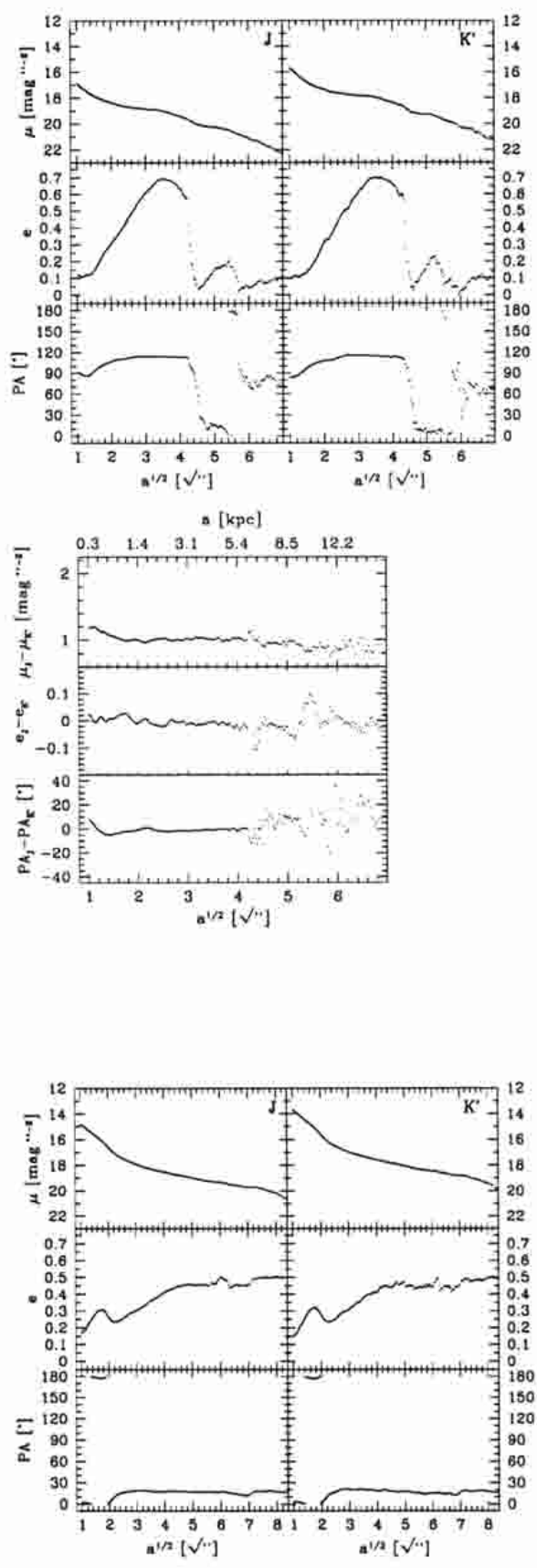

a $[\mathrm{kpc}\}$

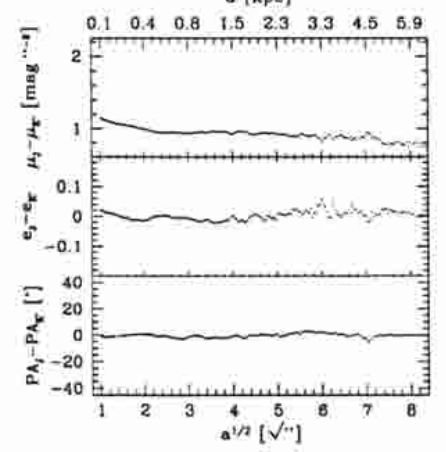

Fig. 2. continued
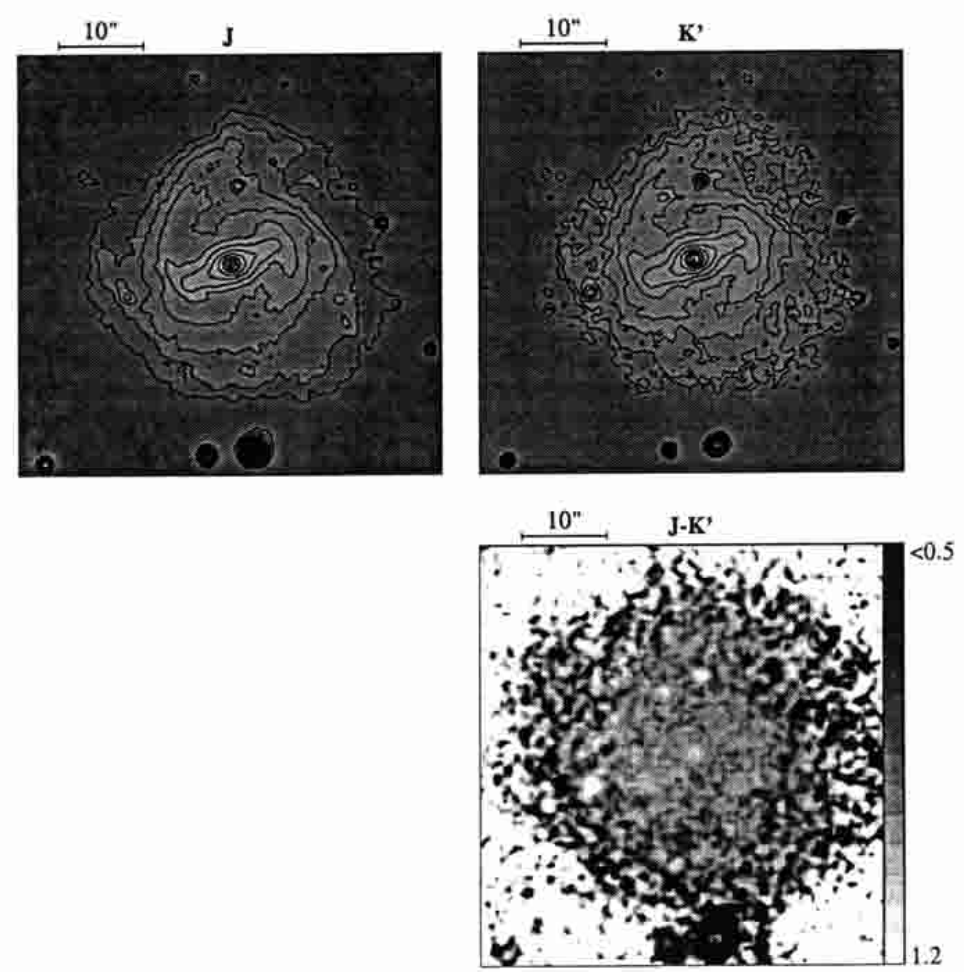

NGC 4941
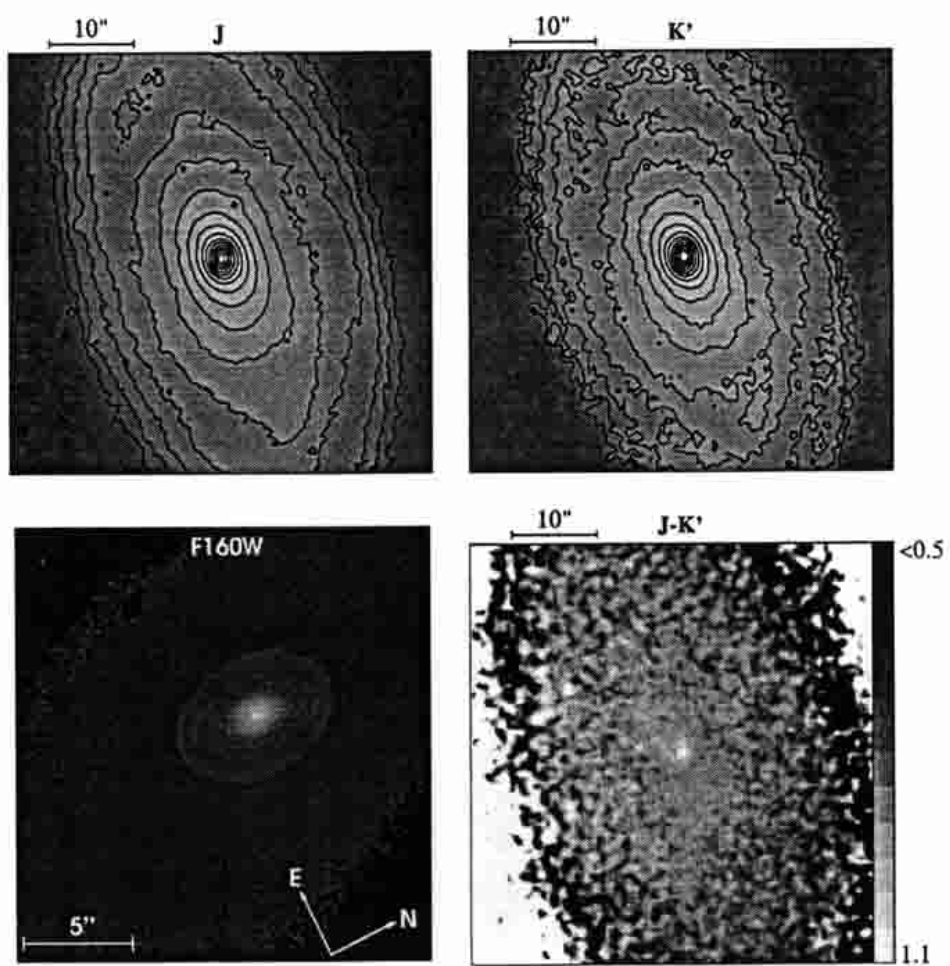
NGC 4939

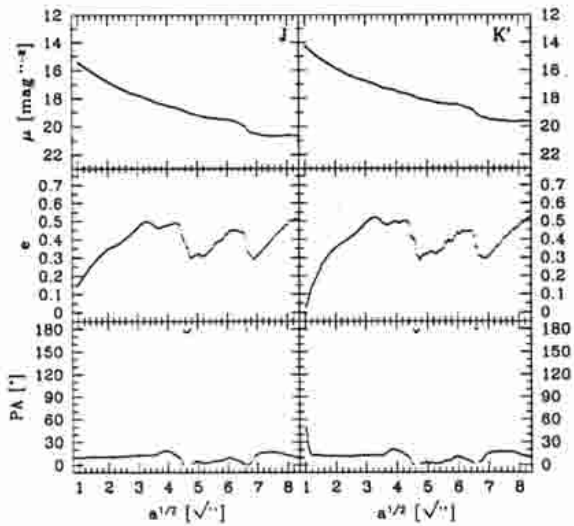

$n$ [kpc]
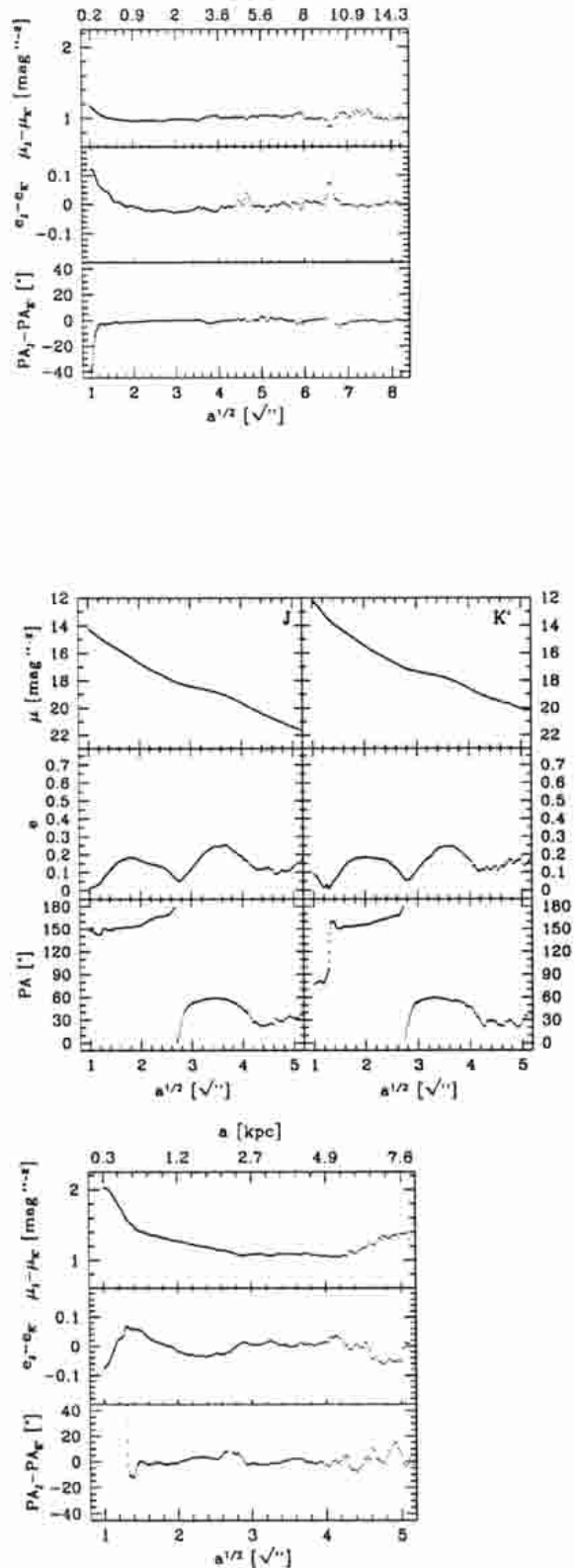
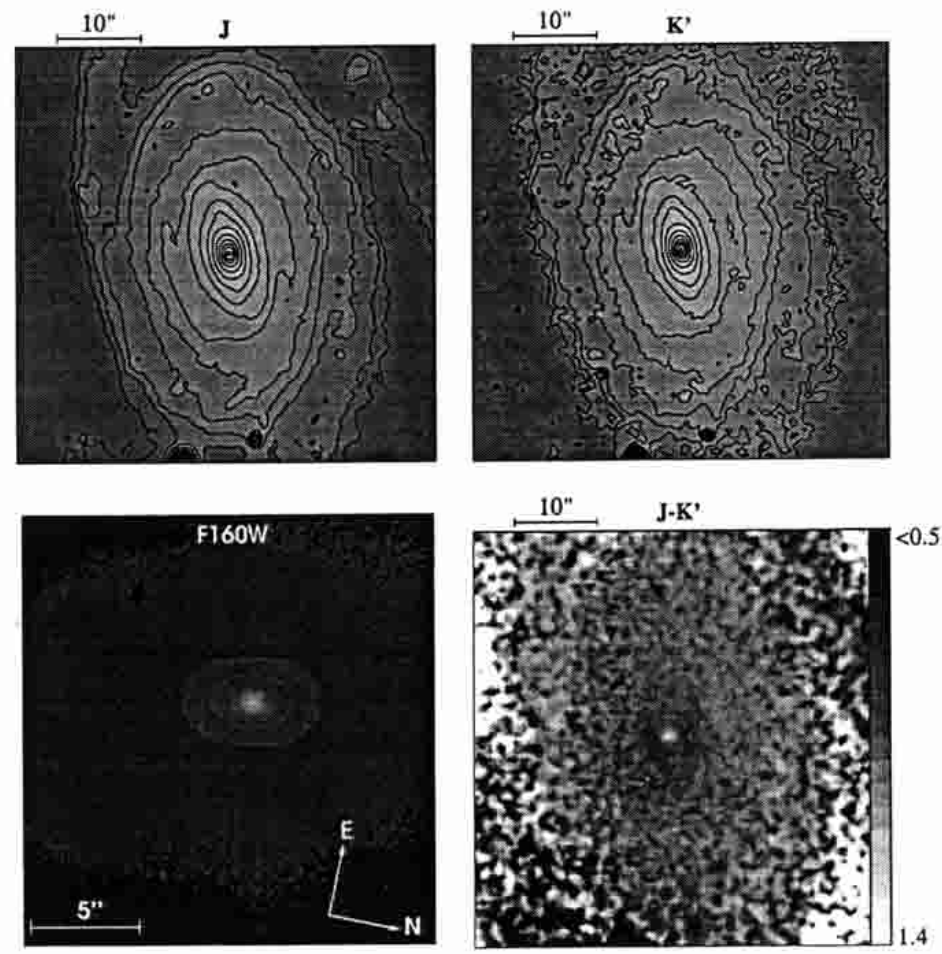

ESO 323-G077
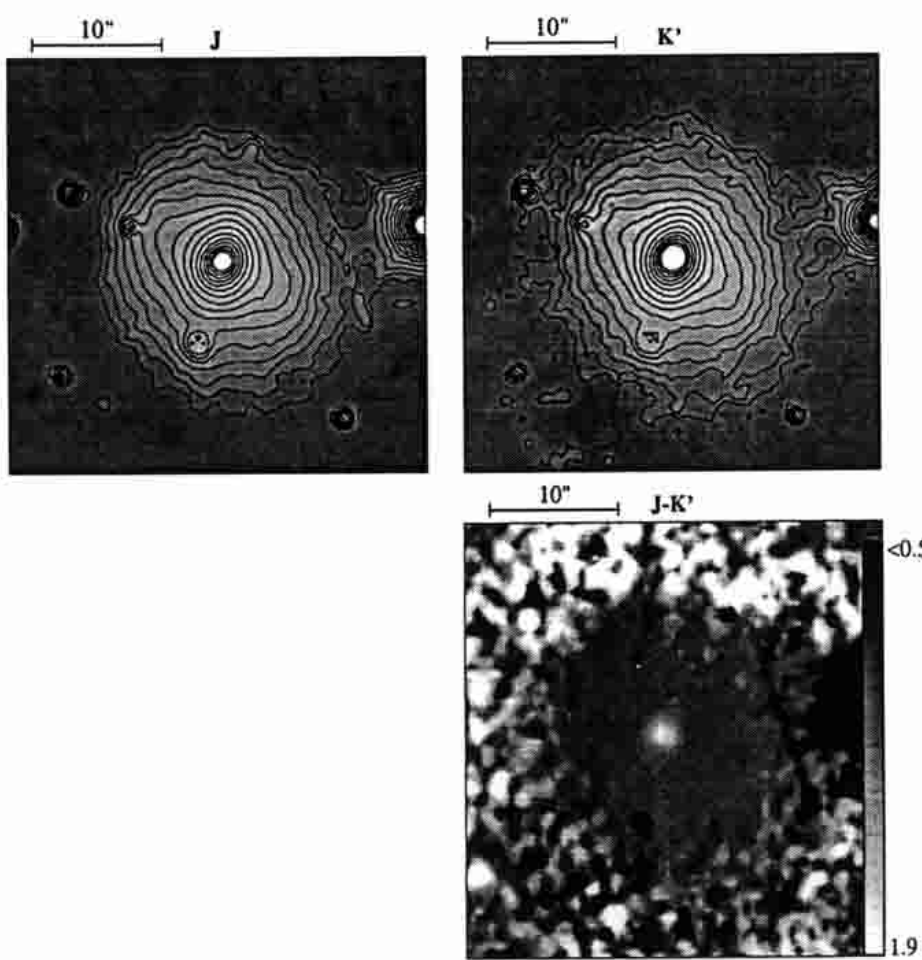

Fig. 2. continued 
ESO 508-G005

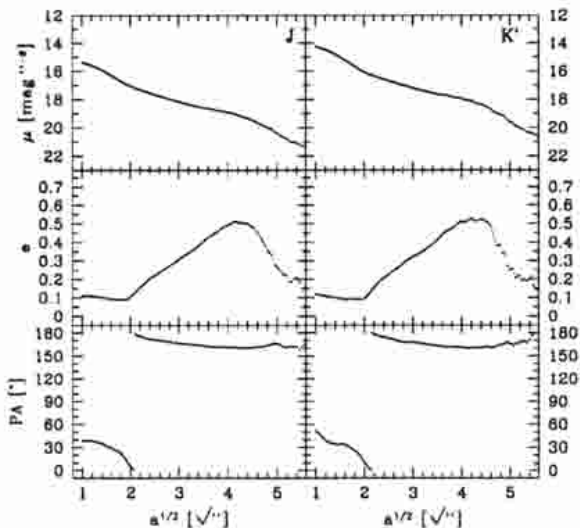

a $[\mathrm{kpc}]$
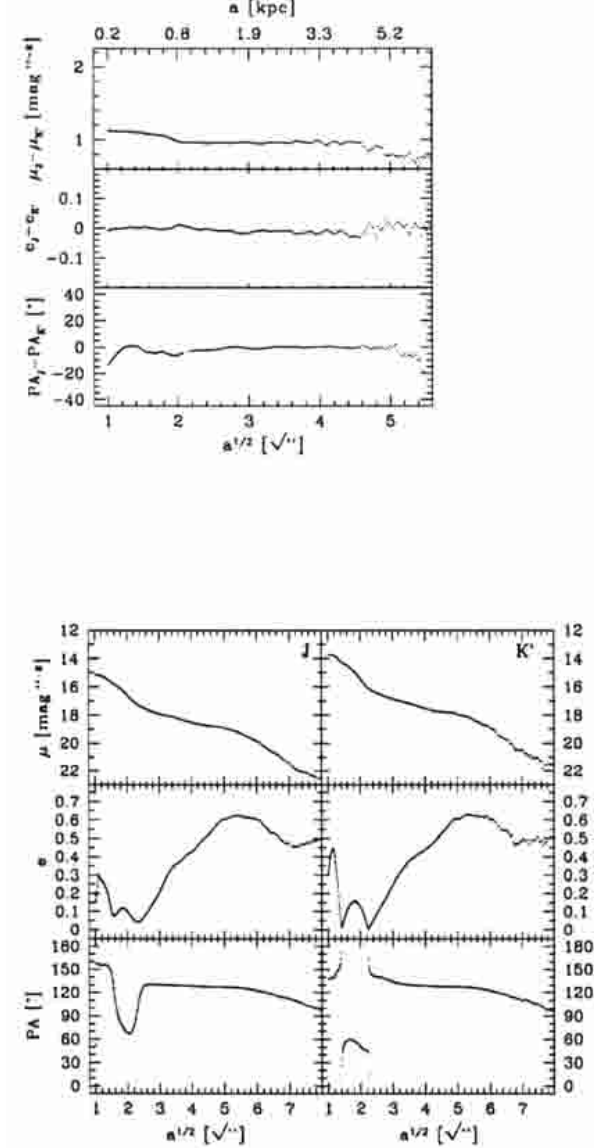

- $[\mathrm{kpc}]$

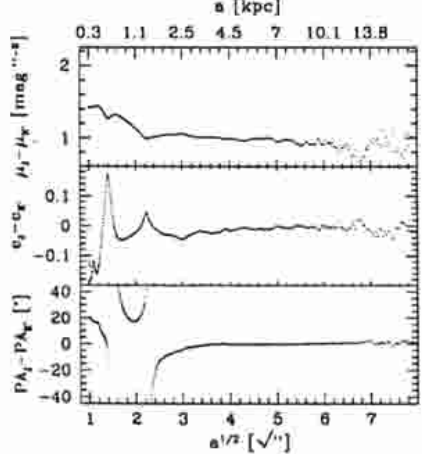

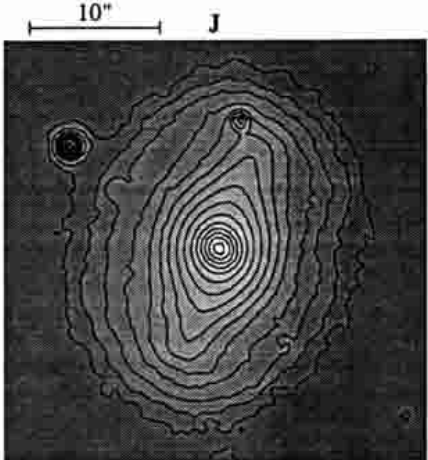
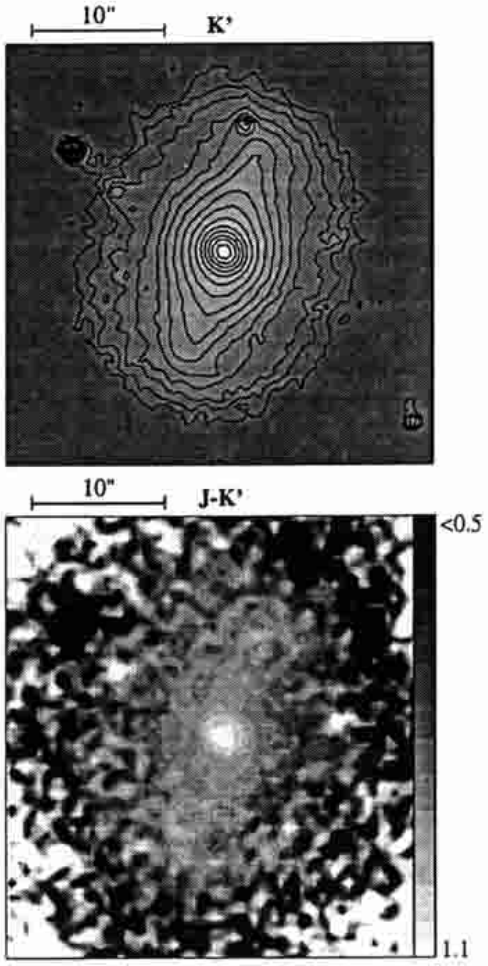

NGC 5135
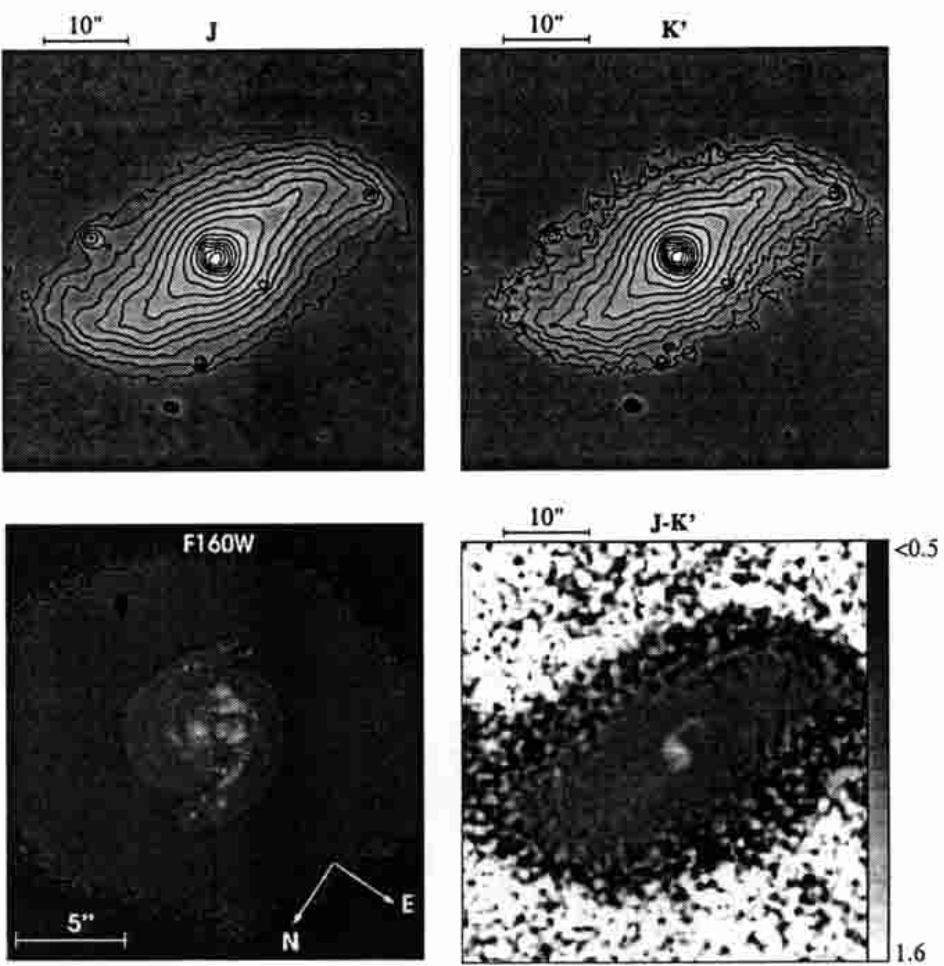

Fig. 2. continued 
NGC 5643

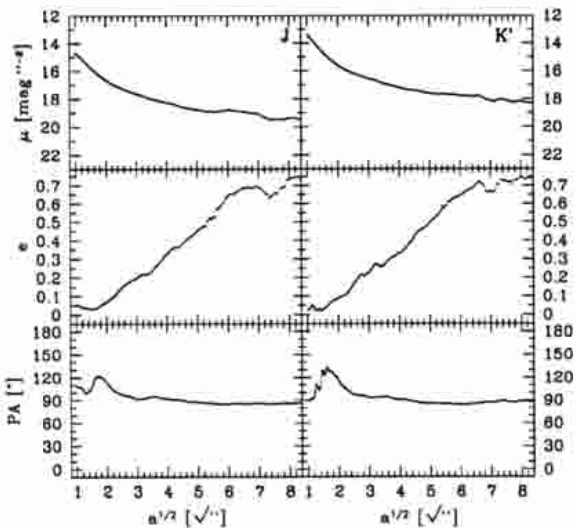

- [ $[\mathrm{kpc}]$
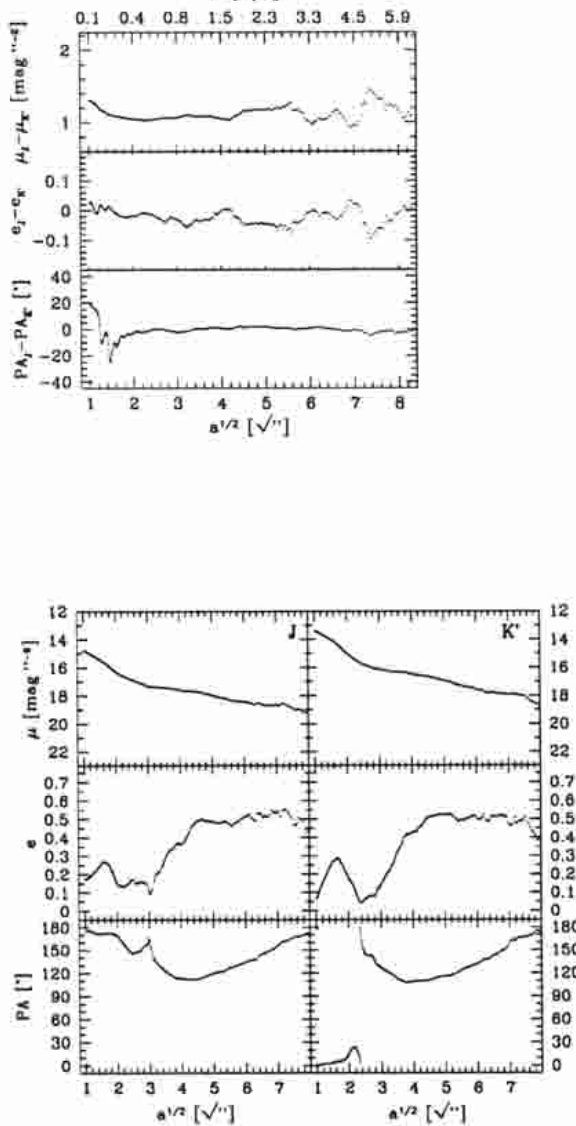

a [kpc]

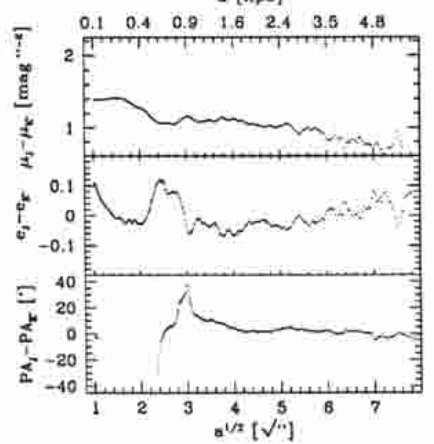

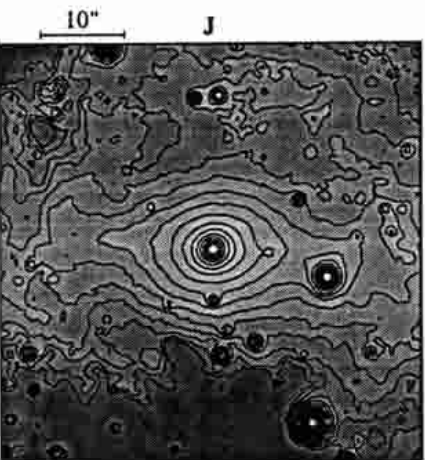
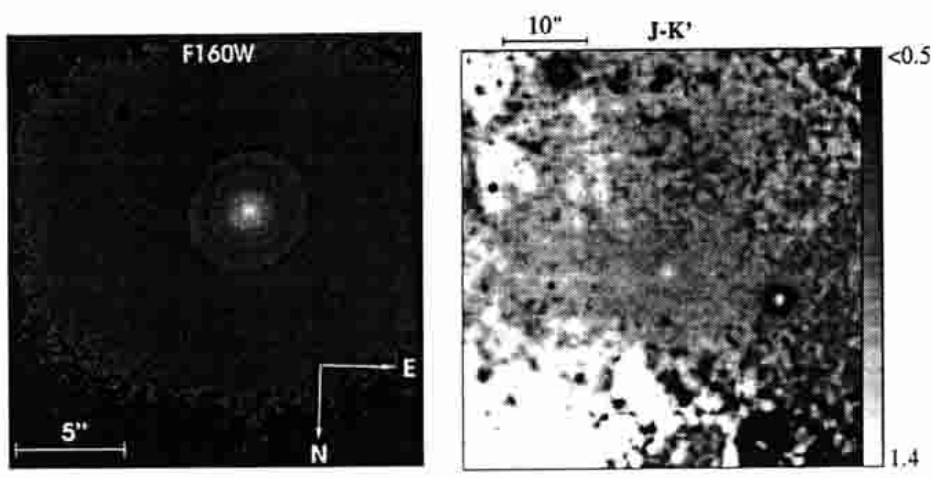

NGC 6221
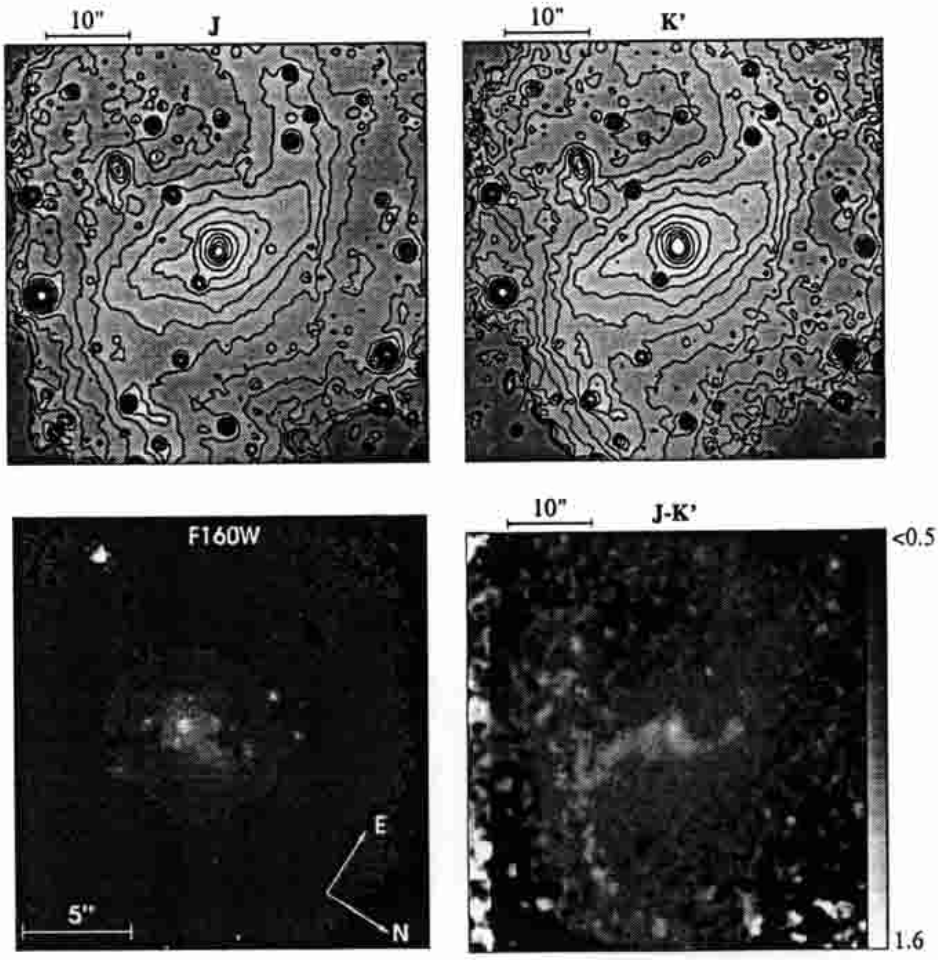

Fig. 2. continued 


\section{NGC 6300}
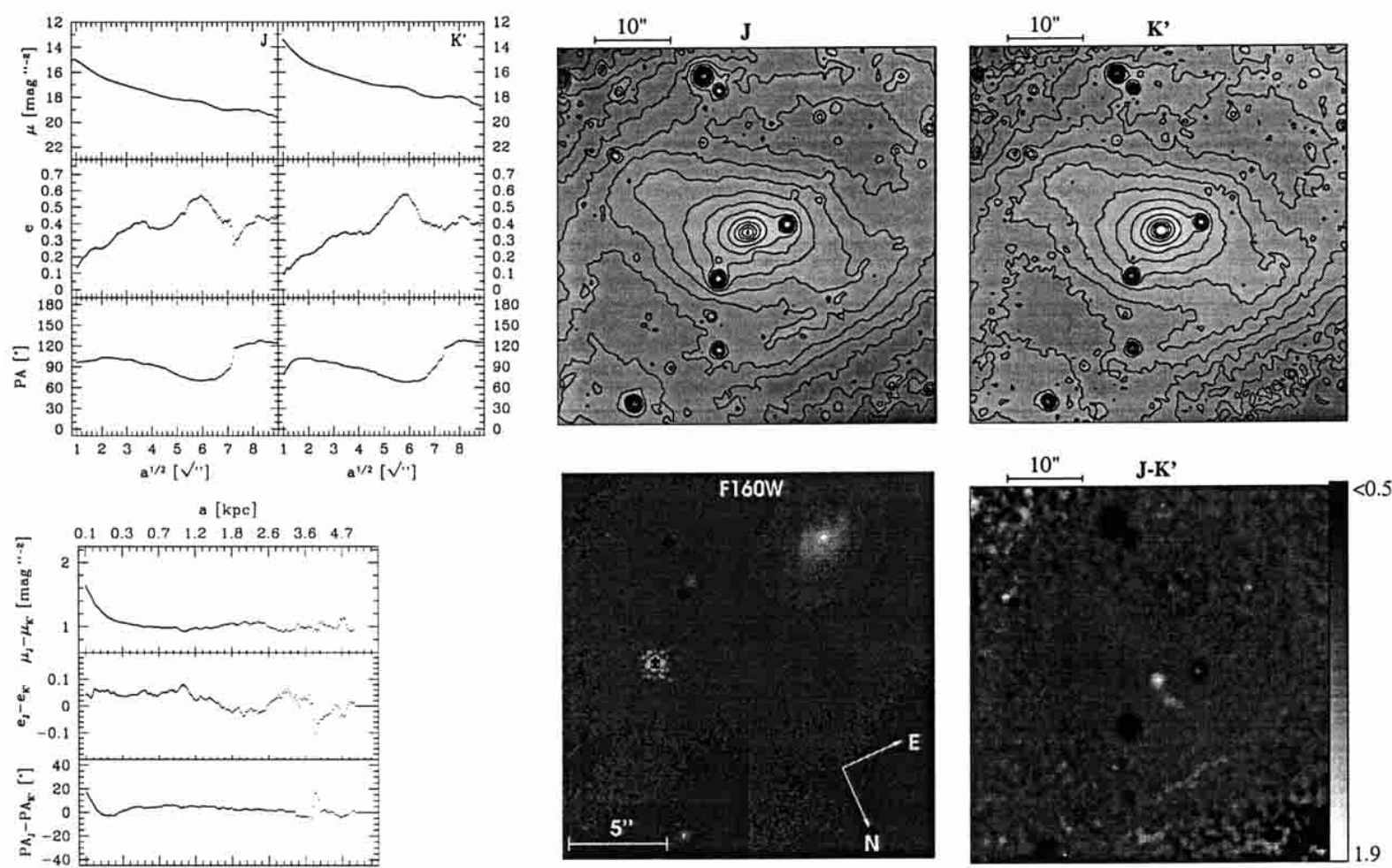

Fig. 2. continued

$4^{\prime \prime}(\approx 2.6 \mathrm{kpc})$. This complex central structure, also appearing in the visible frame of Kazès et al. (1990), may be a late stage merger. This central feature results in a bar-like contour map and ellipticity profiles. But it is interesting to note that the PA does not show any plateau in the region of this bar. Indeed, it decreases almost linearly from the centre up to $\approx 17^{\prime \prime}$. This characteristic could be the signature of the strong interaction, the bar being strongly triaxial and perhaps not coplanar.

\section{2. $E S O 264-G 036\left(S B b, 1^{\prime \prime} \approx 0.45 \mathrm{kpc}\right)$}

This is a single-barred object. The well-defined primary bar ends at $\approx 18^{\prime \prime}$ where a slight twist is observed due to the start of the spiral arms. In the inner region $\left(a \leq 2.5^{\prime \prime}\right)$, the $\mu_{J}-\mu_{K^{\prime}}$ profile steeply decreases from $\approx 1.5 \mathrm{mag}^{\prime \prime}{ }^{-2}$ at the center to $\approx 1.1 \mathrm{mag}^{\prime \prime}-2$. The $e_{J}-e_{K^{\prime}}$ profile is roughly constant at $\approx 0.03$, while the $\mathrm{PA}_{J}-\mathrm{PA}_{K^{\prime}}$ profile increases from about $-10^{\circ}$ to $\approx 5^{\circ}$. For $a \geq 2.5^{\prime \prime}$, the $\mu_{J}-\mu_{K^{\prime}}$ profile slowly increases again up to $\approx 1.4 \mathrm{mag}^{\prime \prime}-2$. This feature corresponds to two red and elongated features, probably star-forming region, close to the bar ends.

\section{3. $N G C 3393\left(S B a b, S T B / S y 2,1^{\prime \prime} \approx 0.23 \mathrm{kpc}\right)$}

We confirm previous observations by Jungwiert et al. (1997) and Alonso-Herrero et al. (1998) that this is a double-barred galaxy. In close agreement with these authors, the primary bar is oriented at $\mathrm{PA}_{\mathrm{p}} \approx 159^{\circ}$, whereas the trailing secondary bar has $\mathrm{PA}_{\mathrm{s}} \approx 145^{\circ}$.

Differential profiles and colour plate show unusual structures: surprisingly, the secondary bar is more prominent on $J$ profiles than on $K^{\prime}$ ones, leading to a small bump in the $e_{J}-e_{K^{\prime}}$ profile. The $\mu_{J}-\mu_{K^{\prime}}$ profile presents an artificial negative gradient in the inner region due to the seeing departure from the two IR bands; this gradient must be an artefact because field stars also have a central blue dip in the $J-K^{\prime}$ colour frame. The same features are reproduced for other objects, when the seeing is artificially degraded in one of the two bands. In Sect. 3.2.2, we use $J$ and $K$-frames from Alonso-Herrero et al. (1998) to avoid this problem.

\section{4. $\operatorname{ESO} 215-G 031\left(S B b, S T B, 1^{\prime \prime} \approx 0.17 \mathrm{kpc}\right)$}

This SBb starburst galaxy can be considered as the prototype of nearly aligned double-barred systems: the ellipticity profile clearly shows two bumps along which 
the position angle is nearly constant in both bands. Those bars also tend to reduce the slope of the $\mu_{J}$ and $\mu_{K^{\prime}}$ profiles along each bar. One of the most striking feature of this object is that the two bars are close to be aligned, $\mathrm{PA}_{\mathrm{p}} \approx 147^{\circ}$ whereas $\mathrm{PA}_{\mathrm{s}} \approx 153^{\circ}$, leading to $\theta_{12} \approx 6^{\circ}$. The ellipticity between those bars declines close to zero at $a \approx 9^{\prime \prime}$, where the PA variation reveals the presence of a nuclear ring. It is observable on the $J-K^{\prime}$ image as well. As this galaxy has both inner and outer rings Buta (1995), this is a three-ringed galaxy.

Three distinct regions of redder $J-K^{\prime}$ colour $\left(\approx 1.3 \mathrm{mag}^{\prime \prime-2}\right)$ are visible on this structure. Two "hotspots" or "twin-peaks" are on the ring and are symmetric with respect to the center, the third one being at the galaxy nucleus. The $\mu_{J}-\mu_{K^{\prime}}$ profile is nearly constant at $1.1 \mathrm{mag}^{\prime \prime-2}$ along the secondary bar and at $1 \mathrm{mag}^{\prime \prime-2}$ beyond. The $e_{J}-e_{K^{\prime}}$ profile shows a slight negative gradient along the secondary bar, and is close to zero elsewhere.

\section{5. $\left.\operatorname{ESO~320-G030~(SABa,~STB,~} 1^{\prime \prime} \approx 0.21 \mathrm{kpc}\right)$}

The visual aspect, confirmed by the profile examination, leads us to classify this galaxy as a new double-barred one. The primary bar has a $\mathrm{PA}_{\mathrm{p}} \approx 142^{\circ}$, different from the $\mathrm{PA}_{\text {disc }}=121^{\circ}$ (see Table 1$)$, so that this objet should be classified as an SB rather than SAB. This bar also presents two regions of slightly higher surface brightness at its ends. The secondary bar has its outermost isophotes twisted, a nearly constant ellipticity, and $\mathrm{PA}_{\mathrm{s}} \approx 107^{\circ}$ (i.e. $\theta_{12} \approx 35^{\circ}$ ).

An extended central structure, clearly visible on the $J-K^{\prime}$ colour map, is aligned with the primary bar. This might be a nuclear ring or disc. Nested at the center of this feature, a region of redder colour $\left(\approx 1.4 \mathrm{mag}^{\prime \prime-2}\right)$ likely corresponds to the secondary bar, but the angular resolution is too poor to reveal unambiguously the bar morphology. The $\mu_{J}-\mu_{K^{\prime}}$ profile decreases from $\approx 1.4 \mathrm{mag}^{\prime \prime-2}$ to reach a constant value $\approx 0.9$ ) beyond $a \approx 9^{\prime \prime}$. The $e_{J}-e_{K^{\prime}}$ and $\mathrm{PA}_{J}-\mathrm{PA}_{K^{\prime}}$ profiles are roughly zero throughout the galaxy, except for the inner region where they amount to $\approx 0.04$ and $\approx 10^{\circ}$ respectively.

\section{6. $\operatorname{ESO~443-G017(SBO/a,STB,1^{\prime \prime }\approx 0.19kpc)}$}

This galaxy presents a primary bar with $\mathrm{PA}_{\mathrm{p}} \approx 12^{\circ}$ that is twisted near its ends as the spiral arms begin to affect the ellipse fitting. The small bump in ellipticity and the constant $\mathrm{PA}_{\mathrm{S}} \approx 31^{\circ}$ suggest the presence of a weak secondary bar. This secondary bar is more pronounced in $K^{\prime}$ profiles than in $J$ ones, resulting in a $e_{J}-e_{K^{\prime}}$ excess in the central region; it is also visible on the $J-K^{\prime}$ colour map. However, the disc inclination and PA (Table 1) prevent any reliable detection from being made. A high inclination angle make it difficult to distinguish between a bar and a projected triaxial structure having the same PA as the disc. The projected angle between both bars would be $\theta_{12} \approx-19^{\circ}$. The $\mu_{J}-\mu_{K^{\prime}}$ profile is nearly constant $\left(\approx 1.3 \mathrm{mag}^{\prime \prime}-2\right)$ along the secondary bar, and then decreases down to a constant value of $\approx 1 \mathrm{mag}^{\prime \prime-2}$ at larger distances.

\section{7. $N G C 4903\left(S B c, S y 2,1^{\prime \prime} \approx 0.31 \mathrm{kpc}\right)$}

A very strong $\left(e^{\max } \approx 0.7\right)$ and quite long $\left(a_{\mathrm{p}} \approx 21^{\prime \prime}\right)$ primary bar is visible. It is surrounded by a weakly barred component whose extent and surface brightness might suggest a lens nature. The central part of this galaxy seems to be relatively featureless, even if the very center shows a constant ellipticity ( $e \approx 0.1$ ) accompanied by a PA variation, in contrast with the profile behaviour at larger radii. This is reinforced by the $\mu_{J}-\mu_{K^{\prime}}$ gradient observed in the same region, which is also visible on the $J-K^{\prime}$ frame. But this region needs a higher angular resolution to be reliably probed.

\section{8. $N G C 4941$ (SABab, Sy2, $\left.1^{\prime \prime} \approx 0.08 k p c\right)$}

The $\mu, e$, and PA profiles all indicate the presence of a nuclear bar, without any large-scale bar. The nuclear bar, clearly visible on the NICMOS F160W frame, is characterized by $\mathrm{PA}_{\mathrm{s}} \approx 180^{\circ}, e_{\mathrm{s}}^{\max } \approx 0.32$, and $a_{\mathrm{s}} \approx 5^{\prime \prime}$. NGC 7702, which also harbour a nuclear bar $\left(e_{\mathrm{s}}^{\max } \approx 0.43, a_{\mathrm{s}} \approx 10^{\prime \prime}\right.$, see Paper I), is visually very close to this galaxy; even the orientation of the bar relative to the disc major axis are similar $\left(\left|\mathrm{PA}_{\mathrm{s}}-\mathrm{PA}_{\mathrm{disc}}\right| \approx 20^{\circ}\right)$.

The $\mu_{J}-\mu_{K^{\prime}}$ profile shows a slight decrease along the nuclear bar and is nearly constant afterwards $\left(\approx 1 \mathrm{mag}^{\prime \prime-2}\right)$.

\section{9. $N G C 4939\left(S A b c, S y 2,1^{\prime \prime} \approx 0.19 k p c\right)$}

The regular decrease of the surface brightness, the roughly constant $\mathrm{PA}$ at the same angle as the disc $\left(\approx 10^{\circ}\right.$, see Table 1), and the high inclination angle of this galaxy, all indicate that the ellipticity behaviour should mostly be due to projection effects of a disc plus spiral arms (see e.g. Jungwiert et al. 1997 for examples of projection effects). Thus, this galaxy really seems unbarred, even if the central isophotes enhanced in the NICMOS F160W frame, have a boxy shape.

Most striking is the big difference in ellipticity between $J$ and $K^{\prime}$ band in the central region $\left(\mu_{J}-\mu_{K^{\prime}} \leq 0.1\right.$ at the center).

\subsection{0. $E S O 323-G 077$ (SBO, STB/Sy1, $\left.1^{\prime \prime} \approx 0.29 \mathrm{kpc}\right)$}

The relatively weak primary bar is oriented at $\mathrm{PA}_{\mathrm{p}} \approx 56^{\circ}$, and ends near $\approx 17.5^{\prime \prime}$. The inner prominent structure has 
Table 3. Non-deprojected morphological parameters in the $K^{\prime}$-band for galaxies with embedded structures

\begin{tabular}{|c|c|c|c|c|c|c|c|c|c|c|}
\hline \multirow[t]{2}{*}{ Galaxies } & \multirow[t]{2}{*}{ Type } & \multicolumn{3}{|c|}{ 1st component } & \multicolumn{3}{|c|}{ 2nd component } & \multicolumn{3}{|c|}{ Ratios } \\
\hline & & $\begin{array}{l}a_{1} \\
{\left[{ }^{\prime \prime}\right]}\end{array}$ & $e_{1}^{\max }$ & $\begin{array}{c}\mathrm{PA}_{1} \\
{\left[^{\circ}\right]}\end{array}$ & $\begin{array}{l}a_{2} \\
{\left[{ }^{\prime \prime}\right]}\end{array}$ & $e_{2}^{\max }$ & $\begin{array}{c}\mathrm{PA}_{2} \\
\left.{ }^{\circ}\right]\end{array}$ & $\beta_{12}$ & $\begin{array}{c}\theta_{12} \\
{\left[^{\circ}\right]} \\
\end{array}$ & $\gamma_{12}$ \\
\hline NGC 3393 & $\mathrm{~B}+\mathrm{B}$ & 19.7 & 0.44 & 159 & 3.5 & 0.12 & 145 & 5.6 & -14 & 2.4 \\
\hline ESO 215-G031 & $\mathrm{B}+\mathrm{B}$ & $\approx 47$ & 0.63 & 147 & 9.5 & 0.48 & 153 & $\approx 4.9$ & 6 & 1.7 \\
\hline ESO 320-G030 & $\mathrm{B}+\mathrm{B}$ & $\approx 37$ & 0.64 & 142 & 5.2 & 0.32 & 107 & $\approx 7.1$ & 35 & 2.1 \\
\hline ESO 443-G017 & $\mathrm{B}+\mathrm{B}$ & 14.7 & 0.48 & 12 & 5.5 & 0.28 & 31 & 2.7 & -19 & 1.5 \\
\hline ESO 323-G077 & $\mathrm{B}+\mathrm{T}$ & 17.5 & 0.25 & 56 & 7.7 & 0.18 & 156 & 2.3 & 100 & 1.2 \\
\hline ESO 508-G005 & $\mathrm{B}+\mathrm{dB} ?$ & 30.9 & 0.53 & 161 & 3.6 & 0.10 & 35 & 8.6 & -54 & 1.8 \\
\hline NGC 5135 & $\mathrm{~B}+\mathrm{nS}$ & 46.1 & 0.63 & 127 & - & - & - & - & - & - \\
\hline NGC 6221 & $\mathrm{~T}+\mathrm{B}$ & $\approx 29$ & 0.53 & 116 & 5.7 & 0.29 & 5 & $\approx 5.1$ & -69 & 3.0 \\
\hline
\end{tabular}

$\approx \leftrightarrow$ eye-based estimation.

$X+Y \leftrightarrow X$ qualifies large-scale structures (1st component), whereas $Y$ stands for small scale ones (2nd component); $\mathrm{B}=$ Barred isophotes, $\mathrm{T}=$ Twisted isophotes, $\mathrm{nS}=$ nuclear Spiral-like feature, $\mathrm{dB}=$ "dissolved Bar".

a moderate ellipticity, and presents a monotonous PA increase from about $150^{\circ}$ to $175^{\circ}$, close to the PA value of the disc (Table 1). These isophote twists certainly indicate the presence of a triaxial bulge. The $\mu_{J}-\mu_{K^{\prime}}$ profile reaches the reddest colour of our sample at the center of the galaxy $\left(\approx 2.2 \mathrm{mag}^{\prime \prime-2}\right)$. This extremely red, roughly axisymmetric region, is striking on the $J-K^{\prime}$ map. Moreover all the differential profiles show strong departure from zero toward the center.

\subsection{1. $\left.\operatorname{ESO~508-G005~(SB0/a,~Sy2,~} 1^{\prime \prime} \approx 0.19 \mathrm{kpc}\right)$}

This galaxy harbours a clear primary bar with $\mathrm{PA}_{\mathrm{p}} \approx 161^{\circ}$. The central region $\left(a \leq 4^{\prime \prime}\right)$ presents a nearly constant and low ellipticity $(e \approx 0.1)$ with a twisted PA around $35^{\circ}$. These profiles clearly suggest the presence of a nuclear component (as to be compared with the profile of a "pure" single-barred galaxy like e.g. ESO 264-G036). Moreover, this region is also slightly redder: the $\mu_{J}-\mu_{K^{\prime}}$ profile displays a central plateau at $\approx 1.1 \mathrm{mag}^{\prime \prime-2}$, whereas beyond another plateau has $\approx 0.9 \mathrm{mag}^{\prime \prime-2}$. A similar feature is observed in the double-barred galaxy ESO 215-G031 and ESO 443-G017!

As $\mathrm{PA}_{\mathrm{s}}$ fairly differs from $\mathrm{PA}_{\text {disc }}$, the central morphology is unlikely to result from the projection of a triaxial bulge. Therefore, an appealing and plausible possibility might be that this component is simply the "remnant" of a secondary bar, or a secondary bar in the process of dissolution (for details see e.g. Friedli 1999). This hypothesis might for instance be checked via stellar and gaseous kinematics to detect fossil signatures of previous potential asymmetries.

\subsection{2. $N G C 5135\left(S B a b, S T B / S y 2,1^{\prime \prime} \approx 0.27 k p c\right)$}

The most striking feature of this galaxy is its very special center. In view of the $\mu, e$, and PA profiles, one could think of a three-component object, i.e. both primary $\left(\mathrm{PA} \approx 130^{\circ}\right)$ and secondary $\left(\mathrm{PA} \approx 140^{\circ}\right)$ bars separated by a triaxial bulge. However, the visual inspection of $J, K^{\prime}$ frames instead suggests the presence of a nuclear bi-symmetric spiral structure nested in the primary bar. This inner component is highlighted on the $J-K^{\prime}$ colour map. It is also visible on the WFPC2 image Malkan et al. (1998 as a dusty and patchy flocculent nuclear spiral. The peculiar nature of this nucleus is strengthened by the highly disturbed differential profiles, which reveal the very unusual differences between $J$ and $K^{\prime}$-bands.

Even on high resolution HST frames, it is very difficult to know if these spiral arms really start from a nuclear bar. Indeed, due to the high amounts of dust and the intense star forming regions, ellipse fits are problematical even on NICMOS F160W images.

\subsection{3. $N G C 5643\left(S A B c, S y 2,1^{\prime \prime} \approx 0.08 k p c\right)$}

Both NIR frames show abnormal structures in the southern region; reflection on a misaligned filter could give rise to such ghosts (none of these structures are visible on the DSS optical frame). So the colour gradient in the $J-K^{\prime}$ colour frame is an artefact. However, as the central region of interest is less affected, ellipse fitting was performed on this area. Following its NIR morphology, we suggest to classify this galaxy as an SB one rather than SAB. The $\mu_{J}-\mu_{K^{\prime}}$ profile is nearly constant $\approx 1 \mathrm{mag}^{\prime \prime-2}$ and presents a slight increase near the center $\left(a \leq 1.1^{\prime \prime}\right)$.

\subsection{4. $N G C$ 6221 (SBbc pec, Sy2, 1" $\approx 0.10 \mathrm{kpc}$ )}

This galaxy may be in weak interaction with NGC 6215 Koribalski (1996a), and with two low-surface brightness galaxies Koribalski (1996b). The classification of this galaxy is somewhat controversial since de Vaucouleurs 
et al. (1991) classify it as a barred spiral, SBc, whereas Sandage \& Tammann (1981) classify it as an ordinary spiral, Sbc. This uncertainty probably comes from the dual nature of this object. Because it exhibits properties of both early and late-type, Vega Beltrán et al. (1998) state that NGC 6221 is an intermediate snapshot of a spiral evolving from early to late-type. Nevertheless near-IR profiles revealed a clear nuclear bar (already mentioned in Forbes \& Norris 1998) nested in a twisted structure. N-body simulations Pfenniger (2000) have shown that such an object could evolve toward a double-barred system as the shape of the large-scale twisted isophotes becoming more and more bar-like after several dynamical time-scale.

Tsvetanov \& Petrosian (1995) have detected 173 H II regions throughout the galaxy. The more intensive ones are mostly located along the primary bar, near the center and along the spiral arms, and are also traced by the big redder structures visible on our $J-K^{\prime}$ colour plate. Thus even if the IRAS indicator does not lead us to classify this object as starburst $\left(\log \left(S_{60} / S_{100}\right) \approx-0.36\right)$, it experiences active star formation.

Vega Beltrán et al. (1998) also detect a ring-like structure of ionized gas with a radius of about $9^{\prime \prime}$. Moreover this ring is related to the presence of an inner Lindblad resonance (ILR) of the primary bar. At this radius the ellipticity effectively reaches a minimum, but no ring is clearly visible on the $J-K^{\prime}$ colour plate.

This galaxy has a $\mu_{J}-\mu_{K^{\prime}}$ profile similar to that of previous objects like ESO 443-G017.

\subsection{5. $N G C 6300\left(S B b, S y 2,1^{\prime \prime} \approx 0.08 k p c\right)$}

Already outlined by Mulchaey et al. (1997), NGC 6300 harbors a primary bar $\left(a_{\mathrm{p}} \approx 50^{\prime \prime}\right)$, whereas we could not confirm the presence of the secondary bar they also suspected. Although this object experiences active star formation like NGC 6221, no strong starburst seems to be active near the central region, except for intense nuclear emission (Crocker et al. 1996 report that H II regions are mostly concentrated in the inner ring at $\left.a \approx 42^{\prime \prime}\right)$. The $\mu_{J}-\mu_{K^{\prime}}$ profile is roughly constant outside the center and is steeply increasing inside $a \lesssim 1.2^{\prime \prime}$.

\section{Global properties and discussion}

\subsection{Near-IR morphologies}

The structural parameters of the 8 galaxies with embedded structures are summarized in Table 3. Obviously no reliable statistics could be performed on such a restricted and biased sample, but it enriches previous studies. Note that morphological parameters are difficult to compare with those of the literature since each author uses various definitions and computation methods. Thus we will make a comparison only with Paper II.

For the B+B category (NGC 3393, ESO 215-G031, ESO 320-G030, ESO 443-G017), ranges of bar lengths and luminosity ratios $\left(2.7 \leq \beta_{12} \leq 7.1,1.5 \leq \gamma_{12} \leq 2.4\right)$ are shifted toward lower value than in Paper II. $\left(4.0 \leq \beta_{12} \leq\right.$ $\left.13.4,1.8 \leq \gamma_{12} \leq 7.5\right)$. Thus our new data tend to decrease the mean $\beta_{12}$ and $\gamma_{12}$ from 7.2 and 3.6 down to 6.5 and 3.0 respectively. Those four double-barred galaxies confirm that no preferential angle are found between the two bars, so that nested bars are dynamically decoupled. Moreover while there is a lack of known objects with low $\theta_{12}$, two galaxies have nearly aligned bars $\left(\left|\theta_{12}\right| \approx 6^{\circ}\right.$ and $\left|\theta_{12}\right| \approx 14^{\circ}$, in ESO 215-G031 and NGC 3393 respectively).

Some galaxies (ESO 264-G036, NGC 3393, NGC 4939, ESO 323-G077, NGC 5135, NGC 6221) exhibits significant differences in their isophote shapes (PA and $e$ ) between the two NIR bands. Quillen et al. (1996) have suggested that non-circular stellar motion and radial colour gradient (resulting from the stellar population gradient), could lead to such ellipticity differences between the two bands. But, in the present case, the ellipticity deviation occurs in the central region where some of the Quillen et al. assumptions are not respected; principally the colour gradient could be strongly affected by dust lanes and/or star formation. Moreover, as we have seen in the case of NGC 3393, isophote shape deviations on small-scale could also result from seeing discrepancy between the two bands.

\subsection{Behaviour of $\mu_{J}-\mu_{K^{\prime}}$ profiles}

Whereas all $\mu_{J}-\mu_{K^{\prime}}$ profiles are roughly constant outside the inner region $\left(\mu_{J}-\mu_{K^{\prime}} \approx 1 \mathrm{mag}^{\prime \prime-2}\right.$ at $\left.R \lesssim 4-5^{\prime \prime}\right)$, all of them increases toward the center or at least are indicative of redder central $J-K^{\prime}$ colour (if we exclude NGC 3393, see Sect. 3.1.3). These $\mu_{J}-\mu_{K^{\prime}}$ profiles are qualitatively and quantitatively very similar to those published in Hunt et al. (1997).

Could the central activities give rise to such central NIR colour profile features? For studying this issue, we have reduced the $\mu_{J}-\mu_{K^{\prime}}$ profile behaviour to one single parameter $\Delta\left(J-K^{\prime}\right)$. It is the difference between the inner and the outer $J-K^{\prime}$ colour. This differential colour has the definite advantage of being independent of the photometric calibration and corrections; in particular it is independent of the airmass, the Galactic extinction or the $K$ correction. The inner $J-K^{\prime}$ colour is defined to be the difference between $\mu_{J}$ and $\mu_{K^{\prime}}$, both integrated within the fitted ellipse $a_{0}=1.5^{\prime \prime}$ semi-major axis. The outer $J-K^{\prime}$ colour is computed between the semi-major axis $a_{1}$ and $1.2 a_{1}$, where $a_{1}=0.1 D_{25}$ and $D_{25}$ is taken from de Vaucouleurs et al. (1993). Thus this outer aperture has a linear spatial extent roughly equivalent for all bright galaxies, regardless to its distance. Concerning 


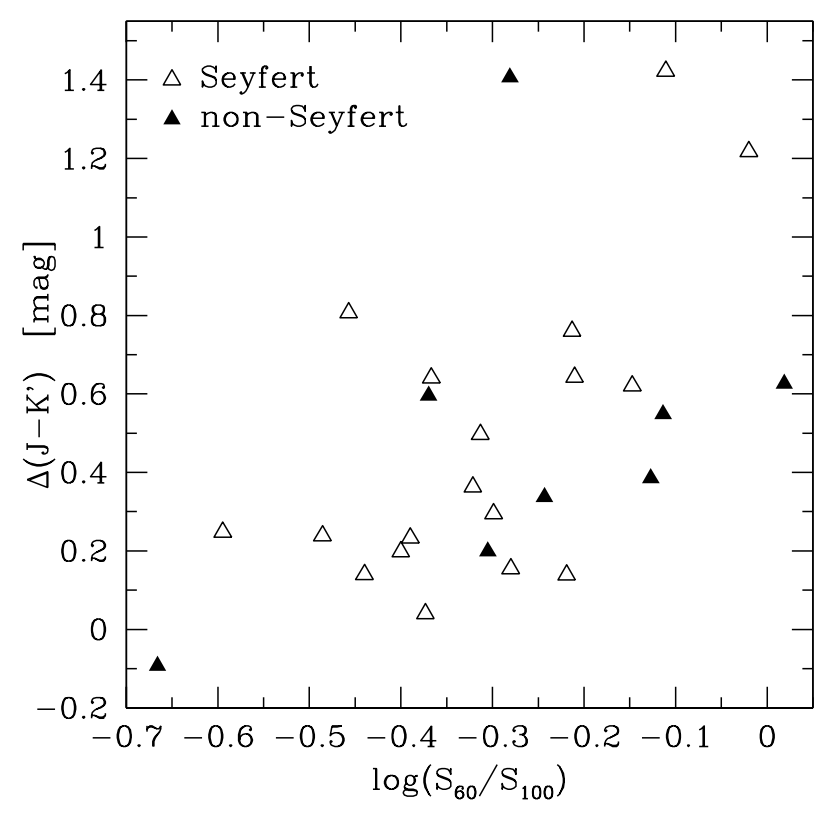

Fig. 3. Central $\mu_{J}-\mu_{K^{\prime}}$ profile behaviour versus star formation intensity (see text). The whole sample is presented in Table 4. Seyfert stands for both Seyfert and/or Liner objects

the inner NIR colour, $a_{0}$ has to be as short as possible in order to probe a region affected as little as possible by activities other than the nuclear ones (e.g. rings of star forming regions). Ideally $a_{0}$ should also be scaled to $D_{25}$ to make it consistent for all our sample, but we consider that the smallest significant aperture must at least have two pixels width ( $\sim a_{0}=1$ pixel). $a_{0}=1.5^{\prime \prime}$ respects this requirement and avoids, for our sample, the problem of contamination by nuclear rings. However, it must be used carefully for more distant objects.

If the central profile behaviour is linked to the Seyfert activity, $\Delta\left(J-K^{\prime}\right)$ should be different for Seyfert and non-Seyfert population. The hypothetic link with starburst activity is less straightforward to enlighten since all disc galaxies are forming stars (and not only in the central region). As the starburst classification is almost arbitrary, we prefer to study the behaviour of $\Delta\left(J-K^{\prime}\right)$ as a function of the star formation rate (SFR). The choice of a SFR estimator is dicussed below.

As the dust absorption peaks in the UV, the dust (mainly the big grains component of Désert et al. 1990 model) in thermal equilibrium with the radiation field reaches a temperature which mostly depends on the UVflux (in fact regardless to the nature of the heating sources). Thus the dust temperature is well suited to study the intensity of the activity which dominates the UVemission. It also has the advantage of probing these source intensities without requiring spectroscopic data which are quite sparse in the archives. In active star-forming galaxies, where UV-emission is dominated by massive stars, this thermal cold dust $(\approx 30 \mathrm{~K}$, Siebenmorgen et al. 1999) mostly peaks around the $50-100 \mu \mathrm{m}$ spectral region 2000 , and references therein. As a consequence, the $60 / 100 \mu \mathrm{m}$ fluxes ratio is very sensitive to the cold dust temperature. Another reason why $\log \left(S_{60} / S_{100}\right)$ may be chosen as tracer of star formation is that, contrary to the mid-IR $(\approx 10-40 \mu \mathrm{m})$, the transiently heated very small grains of dust do not contaminate the $60-100 \mu \mathrm{m}$ cold dust emission Cesarsky \& Sauvage (2000). Unfortunately the low angular resolution of IRAS data $\left(2^{\prime}\right.$ at $\left.100 \mu \mathrm{m}\right)$ only allows a global estimation of the SFR intensity of a galaxy, regardless to its spacial distribution.

In view of the previous considerations, a link between Seyfert and/or starburst activity and NIR colour profile can be probed in the diagram $\Delta\left(J-K^{\prime}\right)$ versus $\log \left(S_{60} / S_{100}\right)$ plotted in Fig. 3. As already mentioned in Sect. 2.4, data from the literature were added in order to obtain more reliable statistics. The whole sample has Hubble Type ranging from $\approx-3$ up to $\approx 7$ (SAB0-SBc), and has its IR properties summarized in Table 4.

As mentionned above, $\Delta\left(J-K^{\prime}\right)$ is independent of the photometric calibrations. Thus its uncertainties mainly come from the readout noise of the detector and are only marginal $(\leq 1 \%)$ compared to the typical conservative error on $\log \left(S_{60} / S_{100}\right)(\approx 10-30 \%)$. This later uncertainty is deduce from the errors on the individual 60 and $100 \mu \mathrm{m}$ IRAS fluxes given in the IRAS Point Source Catalogue (see e.g. Young et al. 1986) which are generally $5-15 \%$.

Independently of the presence of a Seyfert nucleus, the range of $\Delta\left(J-K^{\prime}\right)$ tends to increase from $-0.1-0.9$ up to $0.3-1.5$ as $\log \left(S_{60} / S_{100}\right)$ increases. Despite the efforts we made to extend the sample, it is still not large enough to unambiguously point out a possible correlation in such a plot. Using NIR colour profile tables given in Peletier et al. (1999), the same trend is observed for 29 objects among their Seyfert sample. As we do not have the original NIR data, these new points are not reported in Fig. 3 and cannot be directly compared to ours. But as they could only amplify the scatter of the $\Delta\left(J-K^{\prime}\right)$ vs. $\log \left(S_{60} / S_{100}\right)$ plot, they could be used to quantify the robustness of the observed trend: while the slope and the zero point of the regression remain nearly the same in both cases, the correlation coefficient is found to be 0.56 for the sample plot in Fig. 3, and 0.55 if the 29 Peletier et al. (1999) objets are added. This low but nearly constant value of the correlation coefficient, suggests that the link between $\Delta\left(J-K^{\prime}\right)$ and $\log \left(S_{60} / S_{100}\right)$ is marginally linear but real. Figure 3 looks very similar if one plots either the earliest-types $(T<3)$ or the latest-types $(T \geq 3)$, so that it is not a Hubble sequence effect. Thus the integrated FIR colour is related to the nuclear NIR colour (scaled to the disc colour): significant starburst galaxies have central $J-K^{\prime}$ $0.3-1.5 \mathrm{mag}$ redder than the disc. Hunt et al. (1999) found that Seyfert 1 and nuclear starburst galaxies have the bulge $J-K$ colour 0.1 mag redder than the disc, whereas Seyfert 2 bulges have the same colour as the disc. 
Table 4. Main properties of the sample plotted in Fig. 3

$\begin{array}{ccccc}\text { Galaxies } & \log \left(\frac{S_{60}}{S_{100}}\right) \Delta\left(J-K^{\prime}\right) J-K^{\prime}\left(1.5^{\prime \prime}\right) & \text { Activity } & \text { Nested } \\ & {[\mathrm{mag}]} & {[\mathrm{mag}]} & & \text { Struct. } \\ (1) & (2) & (3) & (4) & (5)\end{array}$

\begin{tabular}{|c|c|c|c|c|c|}
\hline NGC $573^{\mathrm{a}}$ & -0.30 & 0.30 & 1.56 & Sy2 & $\mathrm{B}+\mathrm{B}$ \\
\hline NGC $1068^{a}$ & -0.11 & 1.42 & 2.65 & STB/Sy2 & $\mathrm{B}+\mathrm{nS} ?$ \\
\hline NGC $2110^{\mathrm{a}}$ & -0.15 & 0.62 & 2.03 & STB/Sy2 & \\
\hline NGC $2992^{\mathrm{a}}$ & -0.32 & 0.36 & 1.94 & STB/Sy2 & \\
\hline NGC $3393^{\mathrm{a}}$ & -0.22 & 0.14 & 1.09 & STB/Sy2 & $\mathrm{B}+\mathrm{B}$ \\
\hline $\mathrm{NGC} 4253^{\mathrm{a}}$ & -0.02 & 1.22 & 2.89 & STB/Sy 1.5 & \\
\hline NGC $4388^{a}$ & -0.21 & 0.76 & 2.27 & STB/Sy2 & \\
\hline $\mathrm{NGC} 470^{\mathrm{b}}$ & -0.28 & 1.41 & 1.47 & STB & $\mathrm{B}+\mathrm{T}$ \\
\hline NGC $4314^{\mathrm{b}}$ & -0.30 & 0.20 & 1.20 & STB/LIN & $\mathrm{B}+\mathrm{B}$ \\
\hline NGC $6951^{b}$ & -0.44 & 0.14 & 1.23 & Sy2 & $\mathrm{B}+\mathrm{T}$ \\
\hline NGC $7098^{b}$ & -0.67 & -0.09 & 1.18 & & $\mathrm{~B}+\mathrm{T}+\mathrm{B}$ \\
\hline NGC $7479^{\mathrm{b}}$ & -0.31 & 0.50 & 1.72 & STB/Sy2 & $\mathrm{B}+\mathrm{T}$ \\
\hline $\mathrm{ESO} 215^{\mathrm{c}}$ & -0.24 & 0.34 & 1.26 & STB & $\mathrm{B}+\mathrm{B}$ \\
\hline $\mathrm{ESO} 264^{\mathrm{c}}$ & -0.37 & 0.60 & 1.47 & & \\
\hline $\mathrm{ESO} 320^{\mathrm{c}}$ & -0.11 & 0.55 & 1.34 & STB & $\mathrm{B}+\mathrm{B}$ \\
\hline $\mathrm{ESO} 323^{\mathrm{c}}$ & -0.21 & 0.64 & 1.85 & STB/Sy1 & $\mathrm{B}+\mathrm{T}$ \\
\hline $\mathrm{ESO} 374^{\mathrm{c}}$ & 0.02 & 0.63 & 1.58 & STB & int. \\
\hline $\mathrm{ESO} 443^{\mathrm{c}}$ & -0.13 & 0.38 & 1.34 & STB & $\mathrm{B}+\mathrm{B}$ \\
\hline $\mathrm{ESO} 508^{\mathrm{c}}$ & -0.40 & 0.20 & 1.13 & Sy2 & $\mathrm{B}+\mathrm{dB} ?$ \\
\hline NGC $4903^{\mathrm{c}}$ & -0.39 & 0.23 & 1.17 & Sy2 & \\
\hline NGC $4939^{\mathrm{c}}$ & -0.59 & 0.25 & 1.42 & Sy2 & \\
\hline NGC $4941^{\mathrm{c}}$ & -0.49 & 0.24 & 1.18 & Sy2 & \\
\hline NGC $5135^{\mathrm{c}}$ & -0.28 & 0.15 & 1.22 & STB/Sy2 & $\mathrm{B}+\mathrm{nS}$ \\
\hline NGC $5643^{\mathrm{c}}$ & -0.37 & 0.04 & 1.35 & Sy2 & \\
\hline NGC $6221^{\mathrm{c}}$ & -0.37 & 0.64 & 1.49 & Sy2 & $\mathrm{T}+\mathrm{B}$ \\
\hline NGC $6300^{c}$ & -0.46 & 0.81 & 1.79 & Sy2 & \\
\hline
\end{tabular}

\footnotetext{
${ }^{a}$ Alonso-Herrero et al. (1998).

b Paper II.

c Our sample.

(2): $S_{60}, S_{100}$ are non-colour corrected fluxes in the $60 \mu \mathrm{m}$ and $100 \mu \mathrm{m}$ IRAS-bands, from Catalogued Galaxies + QSOs observed in IRAS Survey, Vers.2 (IPAC 1989),

(3): defined in Sect. 3.2.2,

(4): $J-K^{\prime}$ integrated within the fitted ellipse with a $1.5^{\prime \prime}$ semimajor axis,

(5): type of activity; (Sy, LIN) $=($ Seyferts, LINER $)$ from respective sources $/ \mathrm{STB}=$ starbursts $\leftrightarrow \log \left(S_{60} / S_{100}\right) \gtrsim-0.35$,

(6): nested structures; symbols are defined in Table 3.
}

Of course these results could not directly be compared to ours but both are compatible: as the bulge scale-length is always larger than the inner photometric aperture we used, the contrast between their inner and outer colour naturally tends to be lower than ours.

\subsection{Explanation attempt of the $\Delta\left(J-K^{\prime}\right)$ excess}

The interpretation of the $\Delta\left(J-K^{\prime}\right)$ red excess observed in starburst galaxies is far from being straightforward. But the strong correlation between $\log \left(S_{60} / S_{100}\right)$ and the nuclear $\mathrm{H} \alpha$ luminosity density found among Markarian galaxies in Mazzarella et al. (1991) might be a guideline and suggests that, for their sample, the bulk of FIR luminosity arises from a nuclear starburst (young enough to still ionize the surrounding material).

Could the red nuclear NIR colour we observed result from a recent nuclear starburst? Apart from the usual contribution of the old stellar population, mainly giants, the $J-K^{\prime}$ colour depends at least on three factors $\left.{ }^{1}: 1\right)$ the amount of dust (extinction is higher in $J$ than in $K^{\prime}$ ), 2) the gas and/or dust temperature (James \& Seigar 1999 claimed that hot dust contributes to $K^{\prime}$ ), and 3) the respective contribution of various types of young stars in star forming regions $\left(K^{\prime}\right.$ luminosity increases much more than $J$ one if $K$ supergiants dominate; $J$ increases slightly more than $K^{\prime}$ if OB stars dominate, James \& Seigar 1999). Thus, in luminous and young star-forming regions $J-K^{\prime}$ naturally tends to increase, so that the trend observed in Fig. 3 may indicate that FIR luminosity is essentially produced by a nuclear starburst.

None of the three factors having an effect on $J-K^{\prime}$ could alone be responsible for the whole variations of $\Delta\left(J-K^{\prime}\right)$ through our sample. Indeed, using Leitherer et al. (1999) starburst model, we are unable to explain $J-K^{\prime}$ differences larger than $\approx 0.5$ mag between an old stellar population and a recent starburst. The maximum $\Delta\left(J-K^{\prime}\right)$ we can obtain is $\approx 0.5-0.6$ mag for a continuous SFR of 10 Myr old with a Salpeter IMF and a solar metallicity, and assuming a $J-K^{\prime} \approx 0.5 \mathrm{mag}$ for the old stellar population.

So additional factors that could also locally change $J-K^{\prime}$ have to be considered, for instance: the $150-170 \mathrm{~K}$ gas component associated with AGN and starburst founded with ISO Sturm et al. (1996) tends to increase $J-K^{\prime}$. The non-thermal continuum of PAHs or VSG likely contributes more to the $K^{\prime}-$ band than the thermal emission of the $150-170 \mathrm{~K}$ gas Rouan et al. (1996). The stellar and interstellar metallicity gradient is another parameter, which effect on $\Delta\left(J-K^{\prime}\right)$ could not accurately be estimated without having previously disentangled the age-dust degeneracy.

1 The spectrum of the free-free emission being almost flat in the NIR, its contribution to the $J-K^{\prime}$ colour vanishes. 
Thus, a general explanation about the trend observed in Fig. 3 cannot be stated. It requires a careful study of the gas and dust properties and of the stellar population in individual objects, for which additional data are essential. For that purpose, $\mathrm{H} 2$ and $\mathrm{Br} \gamma$ NIR narrow-band imaging of ESO 215-G031 and NGC 3081, two double-barred galaxies, have already been performed and will be used to carry on our investigations in a forthcoming paper.

\section{Conclusions}

This NIR study on 15 active (starburst and/or Seyfert) objects leads us to these morphological results:

- 4 galaxies are double-barred systems: NGC 3393 (already known), ESO 215-G031, ESO 320-G030, ESO 443-G017 (new ones). These objects confirm previous studies which concluded that embedded bars are dynamically decoupled;

- 4 galaxies have nested feature inside the primary bar: three present a triaxial bulge (ESO 508-G005, ESO 323-G077, NGC 6221), one (NGC 5135) harbours a nuclear spiral structure;

- We suggest to classify NGC 5643 and ESO 320-G030 as $\mathrm{SB}$ rather than $\mathrm{SAB}$;

- Among the 2 unbarred galaxies (NGC 4939, NGC 4941), one (NGC 4941) harbours a nuclear bar (like e.g. NGC 7702, Paper I);

- The Seyfert or starburst activity is not directly linked to the presence of embedded structures: galaxies with embedded structures span a wide range of starburst activity and are found in Seyferts and non-Seyferts (see Fig. 3 and Table 4).

A careful examination has been applied to $\mu_{J}-\mu_{K^{\prime}}$, and has lead us to the following considerations:

- The outer $\mu_{J}-\mu_{K^{\prime}}$ profiles are roughly constant $\left(\approx 0.8-1.2 \mathrm{mag}^{\prime \prime}{ }^{-2}\right)$;

- For the whole sample, the $\mu_{J}-\mu_{K^{\prime}}$ profiles increases toward the center or at least is constant at higher value than the disc;

- We have characterized these inner $\mu_{J}-\mu_{K^{\prime}}$ profiles behaviour by the $\Delta\left(J-K^{\prime}\right)$ parameter which seems to be:

i) independent of the Seyfert activity;

ii) linked to the starburst indicator $\log \left(S_{60} / S_{100}\right)$. Starburst galaxies tend to have a central $J-K^{\prime}$ value $0.3-1.5 \mathrm{mag}$ higher than the disc one. This trend links up the nuclear NIR colour to the integrated FIR colour;

- A young nuclear starburst naturally tends to increase $\Delta\left(J-K^{\prime}\right)$, and could be partially responsible for the trend mentioned above;

- Classical starburst models are unable to reproduce a $\Delta\left(J-K^{\prime}\right) \gtrsim 0.5 \mathrm{mag}$, so that other factors or components have to be considered (e.g. dust extinction, the metallicity or the stellar population gradient, warm gaz). Their quantitative incidence on $\Delta\left(J-K^{\prime}\right)$, requires additional data.

Acknowledgements. This project is supported by the Swiss National Science Foundation (FNS). This research have made use of the NASA/IPAC Extragalactic Database (NED) which is operated by the Jet Propulsion Laboratory, California Institute of Technology, under contact with the U.S. Aeronautics and Space Administration. Partially based on observations made with the NASA/ESA Hubble Space Telescope, obtained from the data archive at the Space Telescope Science Institute. STScI is operated by the Association of Universities for Research in Astronomy, Inc. under NASA contract NAS 5-26555. We warmly thank Almudena Alonso-Herrero for its NIR data.

\section{References}

Alonso-Herrero A., Simpson C., Ward M.J., Wilson A.S., 1998, ApJ 495, 196

Buta R., 1995, ApJS 96, 39

Carter B.S., Meadows V.S., 1995, MNRAS 276, 734

Cesarsky C.J., Sauvage M., 2000, in Block D., Puerari I., Stockton A., Ferreira W. (eds.), "Towards a New Millennium in Galaxy Morphology". Dordrecht, Johannesburg, South Africa (in press)

Crocker D.A., Baugus P.D., Buta R., 1996, ApJS 105, 353

de Vaucouleurs G., 1974, in IAU Symp. 58: The Formation and Dynamics of Galaxies, Vol. 58, p. 335

de Vaucouleurs G., de Vaucouleurs A., Corwin J.R., et al., 1991, in Third reference catalogue of bright galaxies

Désert F.X., Boulanger F., Puget J.L., 1990, A\&A 237, 215

Dultzin-Hacyan D., Benitez E., 1994, A\&A 291, 720

Forbes D.A., Norris R.P., 1998, MNRAS 300, 757

Friedli D., 1999, in Beckman J.E., Mahoney T.J. (eds.), ASP Conf. Ser.

Friedli D., Martinet L., 1993, A\&A 277, 27

Friedli D., Wozniak H., Rieke M., Martinet L., Bratschi P., 1996, A\&AS 118, 461 (Paper II)

Glass I.S., Moorwood A.F.M., 1985, MNRAS 214, 429

Ho L.C., Filippenko A.V., Sargent W.L.W., 1997, ApJ 487, 591

Hunt L.K., Malkan M.A., 1999, ApJ 516, 660

Hunt L.K., Malkan M.A., Moriondo G., Salvati M., 1999, ApJ 510,637

Hunt L.K., Malkan M.A., Salvati M., et al., 1997, ApJS 108, 229

James P.A., Seigar M.S., 1999, A\&A 350, 791

Jarvis B.J., Dubath P., Martinet L., Bacon R., 1988, A\&AS 74,513

Jungwiert B., Combes F., Axon D.J., 1997, A\&AS 125, 479

Kazès I., Proust D., Mirabel L.F., et al., 1990, A\&A 237, L1

Knapen J.H., Shlosman I., Peletier R.F., 2000, ApJ 529, 93

Koribalski B., 1996a, in ASP Conf. Ser. 91: IAU Colloq. 157: Barred Galaxies, p. 172

Koribalski B., 1996b, in ASP Conf. Ser. 106: The Minnesota Lectures on Extragalactic Neutral Hydrogen, p. 238

Leitherer C., Schaerer D., Goldader J.D., et al., 1999, ApJS 123,3

Malkan M.A., Gorjian V., Tam R., 1998, ApJS 117, 25 
Márquez I., Durret F., Delgado R.M.G.l., et al., 1999, A\&AS 140,1

Mazzarella J.M., Bothun G.D., Boroson T.A., 1991, AJ 101, 2034

Mulchaey J.S., Regan M.W., 1997, ApJ Lett. 482, L135

Mulchaey J.S., Regan M.W., Kundu A., 1997, ApJS 110, 299

Peletier R.F., Knapen J.H., Shlosman I., et al., 1999, ApJS 125,363

Pfenniger D., 2000, in Block D., Puerari I., Stockton A., Ferreira W. (eds.), "Towards a New Millennium in Galaxy Morphology". Dordrecht, Johannesburg, South Africa (in press)

Quillen A.C., Ramirez S.V., Frogel J.A., 1996, ApJ 470, 790

Rouan D., Tiphene D., Lacombe F., 1996, A\&A 315, L141

Rowan-Robinson M., Crawford J., 1989, MNRAS 238, 523

Sandage A., Tammann G.A., 1981, A revised Shapley-
Ames Catalog of bright galaxies, Washington: Carnegie Institution, 1981, Preliminary version

Shlosman I., Frank J., Begelman M.C., 1989, Nat 338, 45

Siebenmorgen R., Krügel E., Chini R., 1999, A\&A 351, 495

Sturm E., Lütz D., Genzel R., et al., 1996, A\&A 315, L133

Tsvetanov Z.I., Petrosian A.R., 1995, ApJS 101, 287

Vega Beltrán J.C., Zeilinger W.W., Amico P., et al., 1998, A\&AS 131, 105

Véron-Cetty M.P., Véron P., 1993, A Catalogue of quasars and active nuclei, ESO Scientific Report, Garching: European Southern Observatory (ESO), — c1993, 6th ed.

Wainscoat R.J., Cowie L.L., 1992, AJ 103, 332

Wozniak H., Friedli D., Martinet L., et al., 1995, A\&AS 111, 115 (Paper I)

Young J.S., Schloerb F.P., Kenney J.D., Lord S.D., 1986, ApJ 304, 443 